\title{
Mycobacterium wolinskyi sp. nov. and Mycobacterium goodii sp. nov., two new rapidly growing species related to Mycobacterium smegmatis and associated with human wound infections: a cooperative study from the International Working Group on Mycobacterial Taxonomy
}

\author{
Barbara A. Brown, ${ }^{1}$ Burkhard Springer, ${ }^{3}$ Vincent A. Steingrube, ${ }^{1}$ \\ Rebecca W. Wilson, ${ }^{2}$ Gaby E. Pfyffer, ${ }^{4}$ Maria J. Garcia, ${ }^{5}$ \\ M. Carmen Menendez, ${ }^{5}$ Beatriz Rodriguez-Salgado, ${ }^{5}$ Kenneth C. Jost, Jr, ${ }^{6}$ \\ Sher H. Chiu, ${ }^{6}$ Grace O. Onyi, ${ }^{1}$ Erik C. Böttger ${ }^{3}$ and Richard J. Wallace, Jr ${ }^{1,2}$
}

Author for correspondence: Barbara A. Brown. Tel: + 1903877 7682. Fax: + 19038777652. e-mail: babrown@uthct.edu

1,2 Department of Microbiology ${ }^{1}$ and the Center for Pulmonary and Infectious Disease Control $^{2}$, The University of Texas Health Center at Tyler, 11937 US Hwy 271, Tyler, TX 75708-3154, USA

${ }^{3}$ Institut für Medizinische Mikrobiologie, Medizinische Hochschule Hannover, 30625 Hannover, Germany

4 Swiss National Center for Mycobacteria, Department of Medical Microbiology of the University of Zürich CH-8028 Zürich, Switzerland

5 Departmento de Medicina Preventiva, Facultad de Medicina, Universidad Autonoma de Madrid, Madrid, Spain

6 Texas Department of Health, Austin, TX, USA
Previous investigations demonstrated three taxonomic groups among 22 clinical isolates of Mycobacterium smegmatis. These studies were expanded to 71 clinical isolates, of which 35 (49\%) (group 1) were identical to five ATCC reference strains including the type strain ATCC $19420^{\top}$. Twenty-eight isolates (39\%) were group 2, and eight isolates $(11 \%)$ were group 3 . Isolates of groups 2 and 3 were most often associated with post-traumatic or post-surgical wound infections including osteomyelitis, were susceptible to sulfamethoxazole, amikacin, imipenem and the tetracyclines, variably resistant to clarithromycin, and susceptible (group 1), intermediately resistant (group 2) or resistant (group 3) to tobramycin. The three groups were similar by routine biochemical and growth characteristics, but had different mycolic acid dimethoxy-4-coumarinylmethyl ester elution patterns by HPLC and different PCR-restriction enzyme patterns of a 439 bp fragment of the hsp-65 gene. Group 3 isolates differed from group 1 by 18 bp by 165 rRNA sequencing and exhibited < $25 \%$ homology by DNA-DNA hybridization, being most closely related to Mycobacterium mageritense. The 16S rRNA of group 1 and group 2 isolates differed by only $3 \mathrm{bp}$, but by DNA-DNA hybridization they exhibited only $\mathbf{4 0} \%$ homology. The following names are proposed: Mycobacterium

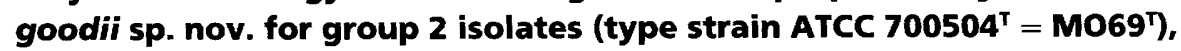
Mycobacterium wolinskyi sp. nov. for group 3 isolates (type strain ATCC

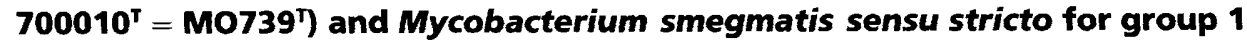
isolates.

Keywords: Mycobacterium goodii, Mycobacterium wolinskyi, Mycobacterium smegmatis, rapidly growing mycobacteria

\section{INTRODUCTION}

Mycobacterium smegmatis was first described in 1885 by Lustgarten as a cause of syphilitic penile ulcers
(Lustgarten, 1885). Although subsequently shown to have no relationship to syphilis, the organism was named for the genital secretions (smegma) from whence it was isolated (Lehmann \& Neumann, 1931). 
The first modern day summary of isolates was in 1953 (Gordon \& Smith, 1953) and described 56 strains, all from culture collections and none from human sources. Several studies (Tsukamura, 1984; Kasatiya et al., 1974) have demonstrated the frequent presence of the organism in environmental sources.

M. smegmatis is currently recognized as an aetiologic agent of bovine mastitis (Richardson, 1970), feline and human post-traumatic wound infections (Plaus \& Hermann, 1991; Richardson, 1970, 1971; Wallace et al., 1988; Wilkinson et al., 1982; Wolinsky \& Rynearson, 1968) and human lung infections (Vonmoos et al., 1986), although for its first 85 years it was regarded as a saprophytic organism of no clinical significance. The first well-described human case involved the lung and pleura in a patient with underlying lipoid pneumonia, and was reported less than 15 years ago (Vonmoos et al., 1986). Since that date there have been a number of well-documented cases of human disease (Plaus \& Hermann, 1991; Wallace et al., 1988).

Although early taxonomic studies of $M$. smegmatis prior to the 1980s suggested the species was homogeneous, these analyses were based on limited phenotypic characteristics and/or involved only a few reference isolates (Kubica et al., 1972; Tsukamura, 1984). Typical tests used to distinguish this species included; a negative $3 \mathrm{~d}$ arylsulfatase reaction, positive iron uptake and positive nitrate reductase reactions, growth at $45^{\circ} \mathrm{C}$ and in the presence of $5 \% \mathrm{NaCl}$, utilization of D-glucitol (D-sorbitol), i-myo-inositol and D-mannitol as sole carbon sources, and late (10-14 d incubation) yellow to orange pigmentation in $50 \%$ of isolates (Gordon \& Smith, 1953; Wayne \& Kubica, 1986; Tsukamura, 1984; Wallace et al., 1988). In subsequent studies in which more recently developed taxonomic techniques were used to evaluate clinical isolates, the heterogeneity among isolates within the species became readily apparent (Steingrube et al., 1995b; Telenti et al., 1993; Wallace et al., 1988; Zhang et al., 1992). This was first observed in a study of 22 clinical isolates of $M$. smegmatis in which three groups (1, 2 and 3 ) were identified based on differences in antibiotic susceptibilities, especially to tobramycin (Wallace et al., 1988).

These interspecies differences were reinforced by genetic methodologies including PCR restriction analysis (PRA) of selected gene sequences. Two studies based on PRA of a $439 \mathrm{bp}$ sequence of the $h s p-65$ gene demonstrated the existence of genetic heterogeneity among isolates of $M$. smegmatis. In the first study, Telenti et al. (1993) reported two isolates of $M$. smegmatis (phenotypic or antibiogram group unknown) that differed from each other as well as from all other mycobacterial taxa with BstEII and HaeIII. Steingrube et al. (1995b) reported a second study that included nine isolates of $M$. smegmatis antibiogram groups 1 and 2 (Wallace et al., 1988) among 129 clinical isolates and reference strains of rapidly growing mycobacteria in which PRA with BstEII, HaeIII,
AciI and $C f o \mathrm{I}$ (isoschizomer of $H h a \mathrm{I}$ ) were compared. These studies found a close relationship between all nine isolates of $M$. smegmatis examined, although antibiogram group 1 and group 2 isolates could be separated with $A c i$.

Heterogeneity of the $\beta$-lactamases extracted from $M$. smegmatis isolates was noted in a study by Zhang et al. (1992). They found two IEF patterns on polyacrylamide gels that were designated type 1 and type 2 . The type $1 \beta$-lactamase pattern was identified in $100 \%$ of 41 isolates belonging to antibiogram group 1 (Wallace et al., 1988), while the type $2 \beta$-lactamase pattern was exhibited by $100 \%$ of 13 isolates of groups 2 and 3 (Wallace et al., 1988).

For the past 19 years, susceptibility testing and/or identification of isolates of rapidly growing mycobacteria has been a primary function of the Mycobacteria/Nocardia laboratory at the University of Texas Health Center at Tyler (UTHCT). Seventyone isolates of $M$. smegmatis were identified during this period. Susceptibility testing produced three different patterns (antibiograms) among these isolates that included varying levels of susceptibility and resistance to tobramycin compared to the findings of Wallace et al. (1988). These groups were designated as M. smegmatis group 1, group 2 and group 3.

These observations prompted a cooperative study of these three groups as well as the newly described species Mycobacterium mageritense (Domenech et al., 1997) by the International Working Group on Mycobacterial Taxonomy (IWGMT). This was a polyphasic analysis, and included standard biochemical analysis, antimicrobial susceptibility testing, GLC, HPLC, PCR restriction analysis of the $65 \mathrm{kDa}$ heat-shock protein gene, 16S rRNA sequencing, 16S RFLP analysis and DNA-DNA hybridization assays following recently presented guidelines for recognition of new mycobacterial species (Levy-Frébault \& Portaels, 1992).

\section{METHODS}

Organisms. Clinical isolates of $M$. smegmatis submitted to the UTHCT Mycobacteria/Nocardia laboratory from 1978 to 1998 were studied. Routine clinical information including culture source was obtained from the referring laboratory at the time each clinical isolate was received. Organisms were stored at $-70^{\circ} \mathrm{C}$ in trypticase soy broth with $15 \%$ glycerol following initial identification and susceptibility testing. Five reference strains of $M$. smegmatis were obtained from the American Type Culture Collection (ATCC; Manassas, VA, USA): the type strain ATCC 19420" ATCC 35797, ATCC 35798, ATCC 14468 and ATCC 607. These five reference strains and 22 of the clinical isolates were partially characterized by Wallace et al. (1988). Five recently characterized strains of $M$. mageritense $(1336,1470,1635,1636$ and the type strain $938^{\mathrm{T}}=$ ATCC $700351^{\mathrm{T}}$ ) (Domenech et al., 1997) were included for comparison with isolates of the $M$. smegmatis groups.

For HPLC analysis, reference strains of Mycobacterium fortuitum ATCC $6841^{\mathrm{T}}$, ATCC 35754 and 170715 , Myco- 
bacterium peregrinum ATCC 14467 $7^{\mathrm{T}}$, ATCC 35755; and I70660; and M. fortuitum third biovariant complex I70868, I70876 and 171013 were used as comparative strains from the $M$. fortuitum complex. These isolates came from the ATCC or from the HPLC Users Group Steering Committee.

For the RFLP analysis of the 16S rRNA genes, control strains included $M$. fortuitum ATCC $6841^{\mathrm{T}}, M$. fortuitum third biovariant ATCC $49403^{\mathrm{T}}$, M. fortuitum third biovariant ATCC $49404^{\mathrm{T}}, \quad$ Mycobacterium parafortuitum strain CNMIVS, M. peregrinum ATCC $14467^{\mathrm{T}}$, Mycobacterium porcinum ATCC $27406^{\mathrm{T}}$, Mycobacterium senegalense NCTC $10956^{\mathrm{T}}$, Mycobacterium chelonae ATCC $35752^{\mathrm{T}}$ and Nocardia asteroides strain CMNIVS.

For the DNA-DNA pairing studies, control strains included $M$. mageritense ATCC $700351^{\mathrm{T}}, M$. smegmatis ATCC $19420^{\mathrm{T}}, M$. fortuitum ATCC $6841^{\mathrm{T}}$ and Mycobacterium phlei IMRU 500 .

Cultural and biochemical characterization. All clinical and reference isolates received prior to 1988 were identified as $M$. smegmatis using standard growth and biochemical methods (Gordon \& Smith, 1953; Kubica et al., 1972; Silcox et al., 1981; Wayne \& Kubica, 1986; Swenson et al., 1985; Tsukamura, 1984). This included typical colony morphology and growth within $7 \mathrm{~d}$ on trypticase soy agar and Middlebrook $7 \mathrm{H} 10$ agar, the absence of early pigmentation (within $7 \mathrm{~d}$ ), positive nitrate reductase and positive ironuptake reactions, a negative $3 \mathrm{~d}$ arylsulfatase reaction, growth at $45^{\circ} \mathrm{C}$, utilization of i-myo-inositol and D-mannitol as sole carbon sources (Silcox et al., 1981; Wallace et al., 1988), and drug susceptibility patterns that included susceptibility to amikacin, ciprofloxacin, doxycycline and sulfamethoxazole, and resistance to cefmetazole and cefoxitin (Wallace et al., 1988). Isolates seen after 1988 were identified on the basis of typical growth and susceptibility patterns, the absence of early pigmentation, a negative $3 \mathrm{~d}$ arylsulfatase reaction, growth at $45^{\circ} \mathrm{C}$, utilization of i-myoinositol and D-mannitol as sole carbon sources, and/or by PRA (Steingrube et al., 1995b; Telenti et al., 1993; Wilson et al., 1998). Additional biochemical features including late pigmentation on $7 \mathrm{H} 10$ agar at $10-14 \mathrm{~d}$, growth on MacConkey agar without crystal violet, degradation of para-aminosalicylate (PAS) to catechol, and utilization of citrate, L-arabinose, D-galactose, D-glucitol (D-sorbitol), Lrhamnose, D-trehalose and D-xylose, as sole carbon sources were determined for selected isolates (Silcox et al., 1981; Tsukamura, 1984). Because all isolates were not tested simultaneously, some biochemical tests were not performed on all isolates.

Susceptibility testing. Susceptibility to nine antimicrobial agents was tested by a broth microdilution method in cationsupplemented Mueller-Hinton broth as previously described (Brown et al., 1992; Swenson et al., 1985). The antimicrobials tested included amikacin, cefmetazole, cefoxitin, ciprofloxacin, clarithromycin, doxycycline, imipenem, sulfamethoxazole and tobramycin. Because all isolates were not tested simultaneously, some isolates were not tested against all of the antimicrobials. Breakpoints used for MICs were those suggested by the National Committee for Clinical Laboratory Standards (NCCLS) (1990) for organisms that grow aerobically, except for cefoxitin, for which an MIC $>32 \mu \mathrm{g} \mathrm{ml}^{-1}$ rather than $>16 \mu \mathrm{g} \mathrm{ml}^{-1}$ was used as the resistance breakpoint.

For taxonomic purposes, susceptibility to tobramycin was established as an MIC of $\leqslant 1 \mu \mathrm{g} \mathrm{ml}^{-1}$, intermediate suscep- tibility as an MIC of 2-8 $\mu \mathrm{g} \mathrm{m}^{-1}$, and resistance as an MIC of $\geqslant 16 \mu \mathrm{g} \mathrm{ml}^{-1}$. Isolates in the susceptible category were designated group 1, those in the intermediate category as group 2, and those that were resistant as group 3 (Wallace $e t$ al., 1988).

Susceptibilities to ethambutol, rifampin and isoniazid were performed by the proportion method on Ogawa egg medium by Tsukamura (1984) and on Mueller-Hinton agar supplemented with OADC (oleate-albumin-dextrose-catalase). Agar disk diffusion (Wallace et al., 1980) with $30 \mu \mathrm{g}$ chloramphenicol per disk and $10 \mu \mathrm{g}$ tobramycin per disk was performed on Mueller-Hinton agar (Becton Dickinson Microbiology Systems) for 40 and 71 isolates, respectively. Thirty-five isolates of $M$. smegmatis group 1, including the five ATCC reference strains, and 28 isolates of $M$. smegmatis group 2 were tested against tobramycin, while $17 \mathrm{M}$. smegmatis group 1 and $15 \mathrm{M}$. smegmatis group 2 isolates were tested against chloramphenicol. All eight isolates of $M$. smegmatis group 3 were tested against both tobramycin and chloramphenicol. Zone sizes were determined after $3 \mathrm{~d}$ incubation at $30^{\circ} \mathrm{C}$. Isolates with zone sizes $>30 \mathrm{~mm}$ for tobramycin were considered to be susceptible; those with 11-30 $\mathrm{mm}$ zone sizes were considered to be of intermediate susceptiblity, and those with zones $\leqslant 10 \mathrm{~mm}$ were considered to be resistant.

\section{HPLC}

Sample processing for fluorescence detection HPLC (FL-HPLC). A culture for FL-HPLC was inoculated onto LöwensteinJensen slants and incubated at $35^{\circ} \mathrm{C}$ for $5 \mathrm{~d}$. A sterile wooden applicator stick was used to transfer a small amount of cell mass to a test tube containing $2.0 \mathrm{ml} 25 \%$ potassium hydroxide in methanol/water $(1: 1, \mathrm{v} / \mathrm{v})$. The tube was capped and autoclaved for $60 \mathrm{~min}$ at $121^{\circ} \mathrm{C}$. After the tube was cooled to room temperature, $1.5 \mathrm{ml}$ chloroform and 1.8 $\mathrm{ml}$ concentrated hydrochloric acid/water $(1: 1, \mathrm{v} / \mathrm{v})$ were added. The tube was vortexed for $60 \mathrm{~s}$ and the layers were allowed to separate. The chloroform layer was transferred to a $13 \times 100 \mathrm{~mm}$ screw cap test tube and evaporated to dryness at $85^{\circ} \mathrm{C}$ at $30 \%$ full vortex speed in a Rapid-Vap (Labconco). One hundred microlitres of $2 \%$ potassium bicarbonate in water/methanol $(1: 1, \mathrm{v} / \mathrm{v})$ were added to the tube and evaporated to dryness at $85^{\circ} \mathrm{C}$. The tube was cooled to room temperature and $100 \mu \mathrm{l}$ chloroform containing $125 \mu \mathrm{g}$ 4-bromomethyl-6-7 dimethoxycoumarin and $15 \mu \mathrm{g} 18$-crown-6 ether (both from Sigma) were added. The tube was sealed with a Teflon-lined cap and heated at $90^{\circ} \mathrm{C}$ for $20 \mathrm{~min}$. After the tube was cooled to room temperature, $1.5 \mathrm{ml}$ chloroform and $1 \mathrm{ml}$ concentrated hydrochloric acid/water/methanol $(1: 1: 2$, by vol.) were added. The tube was vortexed briefly and the layers were allowed to separate. The chloroform layer was transferred to a tube and evaporated. The sample was dissolved in $300 \mu \mathrm{l}$ chloroform that contained $50 \mathrm{ng} \mathrm{ml}^{-1}$ and $200 \mathrm{ng} \mathrm{ml}^{-1}$, respectively, of Low and High Molecular Mass Internal Standards (LMWS and HMWS) for fluorescence-HPLC (Ribi Immunochem Research); $100 \mu$ l of this solution was transferred to an autosampler vial that contained $400 \mu$ chloroform.

FL-HPLC. HPLC was performed with a Hewlett Packard 1050 series pump and autosampler. The pump gradient has been described previously (Butler et al., 1996a). Sample injection volume was $5 \mu \mathrm{l}$. Mycolates were separated with a Beckman $4.6 \times 70 \mathrm{~mm}, 3 \mu \mathrm{m} \mathrm{C}-18$ particle cartridge column (Beckman) maintained at $35^{\circ} \mathrm{C}$. Mycolates were detected with a Jasco FP-920 fluorescence detector (Jasco) equipped 
with a high-sensitivity photomultiplier (gain $=1000)$ and a $16 \mu$ flowcell. Excitation and emission were set at 351 and $430 \mathrm{~nm}$, respectively. Emission bandwidth was $18 \mathrm{~nm}$. The digital filter was used $(S=3)$ and the signal was collected at $2 \mathrm{~Hz}$. M. smegmatis groups 1, 2 and 3 strains were processed and analysed by a standardized UV-absorbance HPLC (UV-HPLC) method (Butler et al., 1996a).

Chromatographic data analysis. Raw FL-HPLC chromatograms were imported into Pirouette Software, version 2.03 (Infometrix). Adjusted retention times (ART) for each chromatogram were derived by alignment of the two internal standards, as has been done previously with mycolic acid $p$ bromophenacyl esters detected by UV absorption (Butler $e t$ al., 1996a, b). Hierarchical cluster analysis was performed on raw normalized chromatographic data from the region between the two internal standards using a mean-centred data preprocess.

GLC. Whole-cell fatty acid analyses were performed by GLC with the Microbial Identification System (MIS; MIDI). The MIS included an HP model 6890A gas chromatograph equipped with a $25 \mathrm{~m} \times 0.2 \mathrm{~mm}$ fused silica capillary column, a flame-ionization detector, an automatic sampler, an integrator and a microcomputer (Hewlett Packard). Peaks were automatically identified and quantified by the system. Organism identification was done by comparison with the MIDI mycobacterial library database (version 3.9). The manufacturer's protocol was followed for all stages of cultivation, extraction, saponification, methylation and chromatography procedures.

Measurement of DNA G + C composition. Mycobacteria were cultured on Löwenstein-Jensen slants. DNA extraction, purification, degradation and $\mathrm{G}+\mathrm{C}$ content determinations by HPLC were performed as previously described by Mesbah et al. (1989), except that a Waters 625 LC System, with a Waters 486 Tunable Absorbance Detector and a Waters 746 Data Module (Millipore/Waters), was used.

PRA of a 439 bp segment of the hsp-65 gene. All isolates of $M$. smegmatis group 3 and $M$. mageritense, and selected isolates of $M$. smegmatis group 1 and $M$. smegmatis group 2 were subjected to PCR amplification of a 439 bp segment of the $h s p-65$ gene as described by Telenti et al. (1993). Cells were grown on tryptic soy agar and ground cell supernatants were used as DNA templates as previously described (Steingrube et al., 1995b; Wilson et al., 1998) using the appropriate positive and negative controls (Telenti et al., 1993).

Screening of multiple commercial restriction endonucleases in previous studies (Steingrube et al., 1995a, b, 1997) resulted in the selection of seven restriction endonucleases (BstEII, HaeIII, HhaI, MspI HinfI, BsaHI and AciI) (New England Biolabs and Promega) for further characterization of isolates. Enzymes were used according to the manufacturer's recommendation, except for $B s a \mathrm{HI}$. To achieve complete digestion with $B s a \mathrm{HI}$, acetylated BSA was substituted for BSA and the digestions mixture was incubated at $60^{\circ} \mathrm{C}$ for $1 \mathrm{~h}$.

Restriction fragments were electrophoresed on 3\% Metaphor agarose ( $4 \mathrm{bp}$ resolution; FMC Bioproducts) containing ethidium bromide $\left(0.625 \mu \mathrm{g} \mathrm{ml}^{-1}\right)$ in a Mini-SubCell electrophoresis system (Bio-Rad) at $95 \mathrm{~V}$ for $1.5-2 \cdot 0 \mathrm{~h}$. Fragment sizes (in bp) were estimated on a computerized Bio Image system (Millipore). Fragments of $60 \mathrm{bp}$ were disregarded as PCR primer artifacts as recommended by Telenti et al. (1993).
165 rRNA sequencing. The 16S rRNA regions chosen for analysis were based on previously published sequence data available in the EMBL/GenBank database (Domenech et al., 1997; Kazda et al., 1992; Kirschner et al., 1992a, b, 1993a, b; Pitulle et al., 1992; Rogall et al., 1990; Stahl \& Urbance, 1990). GenBank accession numbers for selected 16S rRNA sequences used for comparison studies were as follows: $M$. fortuitum ATCC $6841^{\mathrm{T}}$, X52933; $M$. fortuitum third biovariant complex sorbitol-positive ATCC $49403^{\mathrm{T}}$, X65528; $M$. fortuitum third biovariant complex sorbitolnegative ATCC $49404^{\mathrm{T}}$, X65529; Mycobacterium abscessus ATCC $19977^{\mathrm{T}}$, X82235; $M$. chelonae ATCC $35752^{\mathrm{T}}$, X82236; Mycobacterium mucogenicum, X80771; M. smegmatis ATCC 14468, X52922; Mycobacterium mageritense ATCC $700351^{\mathrm{T}}, \mathrm{X} 99938$; and Mycobacterium peregrinum, X52921.

The methods used for DNA extraction, gene fragment amplification of 16S rRNA, and sequence determination have been described previously (Rogall et al., 1982; Böttger, 1989, Böddinghaus et al., 1990; Edwards et al., 1989; Kirschner et al., 1993a, b; Rogall et al., 1990). Briefly, to extract nucleic acids, a colony from a Löwenstein-Jensen slant was suspended in $500 \mu 1$ of $10 \mathrm{mM}$ Tris- $1 \mathrm{mM}$ EDTA (TE). The bacterial pellet was harvested by centrifugation in a microcentrifuge and then dispersed in $100 \mu \mathrm{l}$ of TE containing acid-washed glass beads (diameter, $100 \mu \mathrm{m}$; Sigma). A tissue disintegrator (H. Mickle) was used to disrupt the cells ( $2 \mathrm{~min}$ at the maximum speed). After centrifugation for $5 \mathrm{~min}, 5 \mu$ of the supernatant was used for PCR.

After amplification of the $5^{\prime}$ part of the $16 \mathrm{~S}$ rRNA gene using primers AGAGTTTGATCCTGGCTCAG (positions 8-28) in combination with TGCACACAGGCCACAAGGGA (positions 1046-1026), the nucleic acid sequences of hypervariable regions A and B (Kirschner et al., 1993b) were determined. Based on the grouping determined by this comparison, representative strains were chosen for nearly complete 16S rRNA analysis. The $3^{\prime}$ part of the $16 \mathrm{~S}$ rRNA gene was amplified using primers GTGTGGGTTTCCTTCCTTGG (positions 830-847) and AAGGAGGTGATCCAGCCGCA (positions 1542-1522). Sequence information on nearly the complete 16S rRNA gene was obtained by PCR-mediated Taq-cycle sequencing using an ABI 373 sequencer (Hultman et al., 1989).

The sequences which we obtained were aligned with selected 16S rRNA sequences as described previously (Rogall et al., 1990). For phylogenetic analyses, only those 16S rRNA regions corresponding to Escherichia coli positions 126-554, 894-1082 and 1101-1332 that were available for rapidly growing mycobacterial species were included. Pairwise (Hamming) distances were calculated by weighting nucleotide differences and insertions-deletions equally. A phylogenetic tree was constructed by using the neighbourliness method. (Rogall et al., 1990; Sourdis \& Nei, 1998).

DNA-DNA hybridization experiments. DNA-DNA homology experiments were performed as described previously (Domenech et al., 1997). Genomic DNA $(0.5 \mu \mathrm{g})$ was labelled in vitro using a nick translation labelling kit (Boehringer Mannheim) and $30 \mu \mathrm{Ci} \quad\left[\alpha{ }^{32} \mathrm{P}\right] \mathrm{dCTP}$ (Amersham).

Portions $(1 \mu \mathrm{g})$ of each unlabelled DNA were bound to nylon membrane filters (Amersham) by using alkaline denaturation and UV fixation. Hybridizations were carried out under stringent conditions for $40 \mathrm{~h}$. The filters were then washed and counted with a liquid scintillation counter (Beckman). The relative binding ratios for each strain were 
Mycobacterium wolinskyi sp. nov. and $M$. goodii sp. nov.

calculated from the counts of homologous DNA bound and were expressed as percentages. Control strains included $M$. fortuitum ATCC $6841^{\mathrm{T}}$ and $M$. phlei IMRU 500.

RFLP analysis of the 16S rRNA gene. RFLP analysis of the 16S rRNA gene were performed using genomic DNA digested with the restriction endonucleases Bam $\mathrm{HI}$ and PstI. The digests were separated by electrophoresis on horizontal gel slabs containing $0.75 \%(\mathrm{w} / \mathrm{v})$ agarose, then transferred to nylon membrane filters (Amersham).

An $804 \mathrm{bp}$ fragment of the $16 \mathrm{~S}$ rRNA gene of $M$. fortuitum type strain ATCC $6841^{\mathrm{T}}$ was obtained by PCR and used as a probe. For DNA amplification the R1 and Rc2 oligonucleotide sequences from the Mycobacterium bovis BCG 16S rRNA sequence were used in $50 \mu \mathrm{l}$ amplification mixtures as previously described (Domenech et al., 1997). The cycling profile consisted of 30 cycles of $1 \mathrm{~min}$ at $94^{\circ} \mathrm{C}, 30 \mathrm{~s}$ at $58^{\circ} \mathrm{C}$, and $1 \mathrm{~min}$ at $72^{\circ} \mathrm{C}$, followed by a final incubation at $72^{\circ} \mathrm{C}$ for $5 \mathrm{~min}$. The ribosomal probe was labelled using the Prime$\alpha$-Gene system (Promega) and $20 \mu \mathrm{Ci}\left[\alpha-{ }^{32} \mathrm{P}\right] \mathrm{dCTP}$.

\section{RESULTS}

\section{Organisms}

A total of 71 clinical isolates of $M$. smegmatis, identified during the 19-year study period from 1978 to the present, and five ATCC reference strains were divided into three groups on the basis of susceptibility to tobramycin and PRA (Wallace et al., 1988). Thirtyfive $(49 \%)$ of the clinical isolates and all five ATCC reference strains (including the type strain ATCC $19420^{\text {T }}$ ) were highly susceptible to tobramycin with MICs $\leqslant 1 \mu \mathrm{g} \mathrm{ml}^{-1}$ and disk zones $>30 \mathrm{~mm}$, had a single PRA pattern, and were designated $M$. smegmatis group 1. Twenty-four $(38 \%)$ of the isolates were intermediately susceptible to tobramycin with MICs of 2-8 $\mu \mathrm{g} \mathrm{ml}^{-1}$ and disk zones of $11-30 \mathrm{~mm}$, exhibited a second unique PRA pattern, and were designated as a new species $M$. smegmatis group 2 . Four additional isolates were highly susceptible to tobramycin, but exhibited the second PRA pattern and were included in the latter group. The remaining eight $(11 \%)$ isolates were resistant to tobramycin with MICs $\geqslant 16 \mu \mathrm{g} \mathrm{ml}^{-1}$ and no zones of inhibition by disk diffusion, exhibited a third PRA pattern, and were designated as $M$. smegmatis group 3.

Eight of eight $(100 \%)$ isolates of $M$. smegmatis group 3,22 of $28(79 \%)$ isolates of $M$. smegmatis group 2, and 34 of $37(92 \%)$ isolates of $M$. smegmatis group 1 were recovered from non-pulmonary sources including post-traumatic or post-surgical infections of skin, soft tissue and/or bone. Clinical histories were available for seven of eight $M$. smegmatis group 3 isolates (Table 1) and 24 of 28 isolates of $M$. smegmatis group 2 (Table 2). Six of eight $(75 \%) M$. smegmatis group 3 infections occurred in Texas, while infections with $M$. smegmatis group 2 were more ubiquitous and only 11 of 28 (39\%) occurred in Texas. Of the M. smegmatis group 3 isolates, three were recovered from surgical wound infections while the remaining four came from traumatic wound infections. Multiple positive cultures were recovered from most patients, and $50 \%$ of the wound infections involving $M$. smegmatis group 3 and $67 \%$ of the wound infections involving $M$. smegmatis group 2 were associated with osteomyelitis (Table 1). Community-acquired soft tissue infections due to $M$. smegmatis group 2 were usually osteomyelitis following a motor vehicle accident, often with metal hardware insertion. Pulmonary disease was strongly associated with the histologic finding of lipoid pneumonia, including disease such as achalasia with chronic aspiration (Table 2).

\section{Morphological and biochemical characterization}

Ninety-three per cent of the $M$. smegmatis group 1 isolates and $78 \%$ of the $M$. smegmatis group 2 isolates produced late (10-14 $\mathrm{d}$ incubation period) yellow to

Table 1. Clinical information for the eight patients infected with $M$. smegmatis group 3

\begin{tabular}{|c|c|c|c|c|c|}
\hline Isolate no. & $\begin{array}{l}\text { Patient } \\
\text { age (yrs) }\end{array}$ & Sex & $\begin{array}{l}\text { Geographic } \\
\text { location }\end{array}$ & Source & Disease (reference)* \\
\hline ATCC 700009 & 69 & $\mathrm{~F}$ & Texas & Sternum & $\begin{array}{l}\text { Surgical wound infection, osteomyelitis } \\
\text { (cardiac surgery) (Wallace et al., 1988) }\end{array}$ \\
\hline ATCC $700010^{\mathrm{T}}$ & 35 & $\mathrm{~F}$ & Switzerland & Face & $\begin{array}{l}\text { Surgical wound infection (plastic surgery) } \\
\text { (Pennekamp et al., 1997) }\end{array}$ \\
\hline MO 178 & 9 & M & Texas & Foot & $\begin{array}{l}\text { Cellulitis, osteomyelitis following stepping } \\
\text { on a nail (Wallace et al., 1988) }\end{array}$ \\
\hline MO 472 & 29 & $\mathrm{~F}$ & California & Thigh & Cellulitis, localized abscess following MVA \\
\hline MO 527 & NA & $\mathbf{M}$ & Texas & Axilla & NA \\
\hline MO 817 & 55 & M & Texas & $\begin{array}{l}\text { Arterial/venous } \\
\text { ports }\end{array}$ & $\begin{array}{l}\text { Infected arterial-venous shunt (on } \\
\text { hemodialysis) }\end{array}$ \\
\hline MO $116 \# 2$ & 55 & $\mathrm{~F}$ & Texas & Elbow & $\begin{array}{r}\text { Cellulitis, osteomyelitis following open } \\
\text { fracture (MVA) (Wallace et al., 1988) }\end{array}$ \\
\hline MF1425 & 40 & $\mathrm{~F}$ & Texas & Calf & Cellulitis following local trauma \\
\hline
\end{tabular}

NA, Not available.

* MVA, motor vehicle accident. 
Table 2. Clinical information for 28 patients infected with $M$. smegmatis group 2

\begin{tabular}{|c|c|c|c|c|c|}
\hline Isolate no. & $\begin{array}{l}\text { Patient } \\
\text { age }(y r s)\end{array}$ & Sex & $\begin{array}{l}\text { Geographic } \\
\text { location }\end{array}$ & Source & Diagnosis (reference)* \\
\hline \multicolumn{6}{|c|}{ Community-acquired wound/bone infections } \\
\hline $\begin{array}{l}\text { ATCC 700504 } \\
(\text { MO 69) }\end{array}$ & 22 & $\mathbf{M}$ & Florida & Calcaneus heel & $\begin{array}{l}\text { Osteomyelitis from penetrating trauma } \\
\text { (Wallace } \text { et al., 1988) }\end{array}$ \\
\hline MO 82 & 20 & $\mathrm{~F}$ & Texas & Femur & $\begin{array}{l}\text { Osteomyelitis from open fracture (MVA) } \\
\text { (Wallace } \text { et al., 1988) }\end{array}$ \\
\hline MO 239 & 73 & M & Alabama & Right third toe & Osteomyelitis from stepping on a nail \\
\hline MO 471 & 21 & M & California & Tibia & $\begin{array}{l}\text { Cellulitis/osteomyelitis from puncture } \\
\text { wound (MVA) (Newton et al., 1993) }\end{array}$ \\
\hline MO 116\#1 & 55 & $\mathrm{~F}$ & Texas & Elbow & $\begin{array}{l}\text { Cellulitis/osteomyelitis from open fracture } \\
\text { (MVA) (Wallace et al., 1988) }\end{array}$ \\
\hline MO 648 & 18 & M & Florida & Leg & Infected wound (?osteomyelitis) \\
\hline MO 382 & 30 & $\mathrm{~F}$ & Texas & Tibia & Osteomyelitis following fracture \\
\hline MO 776 & NA & $\mathrm{F}$ & Minnesota & Femur & $\begin{array}{l}\text { Osteomyelitis post-surgical (hardware) } \\
\text { replacement }\end{array}$ \\
\hline MO 881 & 26 & $\mathrm{~F}$ & California & Left thigh, femur & $\begin{array}{l}\text { Cellulitis/osteomyelitis from an open } \\
\text { avulsion injury (MVA) (Newton et al., } \\
\text { 1993) }\end{array}$ \\
\hline MO 65 & 12 & $\mathbf{M}$ & Texas & Cheek & Cellulitis (MVA) (Wallace et al., 1988) \\
\hline MO 947 & 16 & M & Russia & Femur & $\begin{array}{l}\text { Osteomyelitis from open fracture (MVA) } \\
\text { hardware insertion }\end{array}$ \\
\hline MO 541 & 13 & M & Utah & Thigh & $\begin{array}{l}\text { Cellulitis/chronic draining sinus from } \\
\text { puncture wound with a nail }\end{array}$ \\
\hline \multicolumn{6}{|c|}{ Nosocomial disease } \\
\hline MO 80 & 64 & M & Texas & Blood & $\begin{array}{l}\text { Intravenous catheter sepsis (Wallace et al., } \\
\text { 1988) }\end{array}$ \\
\hline MO 143 & 62 & M & Texas & Sternum & Cardiac bypass infection (osteomyelitis) \\
\hline MO 793 & 26 & $\mathrm{~F}$ & Oklahoma & Right breast & Infection following breast reduction surgery \\
\hline MO 730 & 20 & $\mathrm{~F}$ & Missouri & Pacemaker & Infected pacemaker site \\
\hline MO 850 & 91 & $\mathrm{~F}$ & California & Pacemaker & Infected pacemaker site \\
\hline MO 57 & 60 & M & California & Sternum & $\begin{array}{l}\text { Wound infection (?osteomyelitis) (Wallace } \\
\text { et al., 1988) }\end{array}$ \\
\hline \multicolumn{6}{|c|}{ Respiratory disease } \\
\hline MO 123 & 58 & M & Australia & $\begin{array}{l}\text { Pleural fluid, } \\
\text { lung biopsy }\end{array}$ & Lipoid pneumonia (Wallace et al., 1988) \\
\hline MO 584 & 76 & M & Oklahoma & $\begin{array}{l}\text { Open biopsy of } \\
\text { lung mass }\end{array}$ & Chronic granulomatous disease \\
\hline MO 815 & 56 & M & Texas & Bronchial wash & Necrotizing granulomatous pneumonia \\
\hline MO 375 & 34 & $\mathrm{~F}$ & Texas & Open lung biopsy & $\begin{array}{l}\text { Bilateral lipoid pneumonia with prior } \\
\text { gastrectomy }\end{array}$ \\
\hline MO 386 & 18 & $\mathbf{M}$ & Indiana & $\begin{array}{l}\text { Bilateral open } \\
\text { lung biopsy }\end{array}$ & Exogenous lipoid pneumonia (mineral oil) \\
\hline MO 659 & 53 & $\mathbf{M}$ & Ohio & Sputum & Achalasia with pulmonary infiltrates \\
\hline \multicolumn{6}{|c|}{ Diagnosis unknown } \\
\hline MO 288 & 90 & $\mathbf{M}$ & Texas & Cerebrospinal fluid & NA \\
\hline MO 302 & 40 & M & Texas & Right thigh tissue & $\mathrm{NA}$ \\
\hline MO 709 & 69 & M & Massachusetts & Knee aspirate & NA \\
\hline MO 547 & 18 & $\mathrm{~F}$ & Texas & Arm & $\mathrm{NA}$ \\
\hline
\end{tabular}

NA, Not available.

* MVA, motor vehicle accident.

orange pigmentation in contrast to isolates of $M$. smegmatis group 3 and $M$. mageritense that produced no pigment (Table 3 ).
The clinical and reference isolates of $M$. smegmatis group 1, the clinical isolates of $M$. smegmatis group 2, and the clinical isolates of $M$. smegmatis group 3 all 
Table 3. Growth characteristics, biochemical features, $\beta$-lactamase patterns, and susceptibility features of $M$. smegmatis groups 1,2 and 3

Some data are from a previous study (Wallace et al., 1988).

\begin{tabular}{|c|c|c|c|c|c|c|c|c|c|}
\hline \multirow[t]{2}{*}{ Feature } & \multicolumn{4}{|c|}{ M. smegmatis group 1} & \multicolumn{2}{|c|}{ M. smegmatis group 2} & \multicolumn{3}{|c|}{ M. smegmatis group 3} \\
\hline & All isolates & $\begin{array}{l}\text { ATCC } \\
19420^{T}\end{array}$ & $\begin{array}{l}\text { ATCC } \\
700011\end{array}$ & $\begin{array}{l}\text { ATCC } \\
700012\end{array}$ & All isolates & $\begin{array}{c}\text { ATCC } \\
\mathbf{7 0 0 5 0 4 ^ { T }}\end{array}$ & All isolates & $\begin{array}{l}\text { ATCC } \\
700009\end{array}$ & $\begin{array}{c}\text { ATCC } \\
700010^{\mathrm{T}}\end{array}$ \\
\hline Smooth colony & $76 \%(25 / 33)$ & - & + & + & $93 \%(26 / 28)$ & + & $100 \%(8 / 8)$ & + & + \\
\hline Growth at $45^{\circ} \mathrm{C}$ at $3 \mathrm{~d}$ & $100 \%(29 / 29)$ & + & + & + & $100 \%(23 / 23)$ & + & $100 \%(8 / 8)$ & + & + \\
\hline Late pigmentation $(>10 \mathrm{~d})$ on $7 \mathrm{H} 10 \mathrm{agar}$ & $95 \%(18 / 19)$ & + & + & + & $78 \%(18 / 23)$ & + & $0 \%(0 / 8)$ & - & - \\
\hline $3 \mathrm{~d}$ arylsulfatase & $5 \%(1 / 21)$ & - & - & - & $5 \%(1 / 21)$ & - & $0 \%(0 / 8)$ & - & - \\
\hline Degradation of $p$-aminosalicylate & $0 \%(0 / 13)$ & - & - & - & $100 \%(8 / 8)$ & + & $100 \%(3 / 3)$ & + & ND \\
\hline $\begin{array}{l}\text { Growth on MacConkey agar (no crystal } \\
\text { violet) }\end{array}$ & $100 \%(15 / 15)$ & + & + & + & $100 \%(13 / 13)$ & + & $100 \%(8 / 8)$ & + & ND \\
\hline Nitrate reduction & $92 \%(12 / 13)$ & + & - & + & $100 \%(16 / 16)$ & + & $100 \%(8 / 8)$ & + & + \\
\hline Iron uptake & $67 \%(10 / 15)$ & + & + & + & $100 \%(17 / 17)$ & + & $100 \%(8 / 8)$ & + & + \\
\hline Growth on $5 \% \mathrm{NaCl}$ & $100 \%(15 / 15)$ & + & + & + & $100 \%(18 / 18)$ & + & $88 \%(7 / 8)$ & + & + \\
\hline \multicolumn{10}{|l|}{ Semiquantitative catalase } \\
\hline$<45 \mathrm{~mm}$ & $87 \%(13 / 15)$ & + & + & + & $89 \%(14 / 16)$ & + & $50 \%(4 / 8)$ & + & + \\
\hline$<60 \mathrm{~mm}$ & $100 \%(11 / 11)$ & + & + & + & $100 \%(11 / 11)$ & + & $100 \%(8 / 8)$ & + & + \\
\hline $68^{\circ} \mathrm{C}$ catalase & $0 \%(0 / 15)$ & ND & ND & - & $7 \%(1 / 14)$ & - & $100 \%(8 / 8)$ & + & + \\
\hline \multicolumn{10}{|l|}{ Utilization of carbon sources: } \\
\hline L-Arabinose & $100 \%(20 / 20)$ & + & + & + & $100 \%(18 / 18)$ & + & $63 \%(5 / 8)$ & + & + \\
\hline Citrate & $20 \%(4 / 20)$ & + & - & - & $26 \%(5 / 19)$ & - & $50 \%(4 / 8)$ & - & + \\
\hline D-Galactose & $80 \%(8 / 10)$ & + & + & - & $42 \%(5 / 12)$ & - & $88 \%(7 / 8)$ & + & + \\
\hline D-Glucitol (D-sorbitol) & $100 \%(17 / 17)$ & + & + & + & $100 \%(18 / 18)$ & + & $100 \%(8 / 8)$ & + & + \\
\hline D-Mannitol & $100 \%(23 / 23)$ & + & + & + & $100 \%(21 / 21)$ & + & $100 \%(8 / 8)$ & + & + \\
\hline i-myo-Inositol & $100 \%(23 / 23)$ & + & + & + & $100 \%(21 / 21)$ & + & $100 \%(8 / 8)$ & + & + \\
\hline L-Rhamnose & $100 \%(14 / 14)$ & + & + & + & $100 \%(16 / 16)$ & + & $100 \%(8 / 8)$ & + & + \\
\hline D-Trehalose & $60 \%(6 / 10)$ & + & + & - & $50 \%(8 / 16)$ & - & $88 \%(7 / 8)$ & + & + \\
\hline D-Xylose & $100 \%(20 / 20)$ & + & + & + & $100 \%(18 / 18)$ & + & $63 \%(5 / 8)$ & + & + \\
\hline \multicolumn{10}{|l|}{$\beta$-Lactamase pattern* } \\
\hline pl $4 \cdot 4$ & $100 \%(40 / 40)$ & + & + & + & $0 \%(0 / 6)$ & - & $0 \%(0 / 3)$ & - & ND \\
\hline pl $4 \cdot 8,4 \cdot 6$ & $0 \%(0 / 40)$ & - & - & - & $100 \%(6 / 6)$ & + & $100 \%(3 / 3)$ & + & $\mathrm{ND}$ \\
\hline \multicolumn{10}{|l|}{ Disk diffusion susceptibility } \\
\hline \multicolumn{10}{|l|}{ Tobramycin $(10 \mu \mathrm{g}$ disk $)$} \\
\hline No zone $(6 \mathrm{~mm})$ & $0 \%(0 / 35)$ & - & - & - & $0 \%(0 / 28)$ & - & $100 \%(8 / 8)$ & + & + \\
\hline $11-30 \mathrm{~mm}$ & $3 \%(1 / 35)$ & - & - & - & $86 \%(24 / 28)$ & + & $0 \%(0 / 8)$ & - & - \\
\hline$>30 \mathrm{~mm}$ & $97 \%(34 / 35)$ & + & + & + & $14 \%(4 / 28)$ & - & $0 \%(0 / 8)$ & - & - \\
\hline \multicolumn{10}{|l|}{ Chloramphenicol ( $30 \mu \mathrm{g}$ disk) } \\
\hline$\geqslant 10 \mathrm{~mm}$ & $100 \%(17 / 17)$ & + & + & + & $100 \%(15 / 15)$ & + & $50 \%(4 / 8)$ & - & + \\
\hline \multicolumn{10}{|l|}{ MIC determinations } \\
\hline \multicolumn{10}{|l|}{ Tobramycin } \\
\hline $\mathrm{MICs} \leqslant 1 \mu \mathrm{g} \mathrm{ml}^{-1}$ & $100 \%(28 / 28)$ & + & + & + & $19 \%(5 / 27)$ & - & $0 \%(0 / 8)$ & - & - \\
\hline $\operatorname{MICs} 2-8 \mu \mathrm{g} \mathrm{ml}^{-1}$ & $0 \%(0 / 28)$ & - & - & - & $81 \%(22 / 27)$ & - & $100 \%(8 / 8)$ & - & - \\
\hline \multicolumn{10}{|c|}{ Antituberculous drug susceptibilities (Ogawa media) } \\
\hline Ethambutol $\left(5 \mu \mathrm{g} \mathrm{ml}^{-1}\right)$ & $100 \%(11 / 11)$ & + & + & + & $100 \%(8 / 8)$ & - & $100 \%(3 / 3)$ & ND & ND \\
\hline Rifampin $\left(25 \mu \mathrm{g} \mathrm{ml}^{-1}\right)$ & $0 \%(0 / 11)$ & - & - & - & $0 \%(0 / 8)$ & - & $0 \%(0 / 3)$ & ND & ND \\
\hline lsoniazid $\left(10 \mu \mathrm{g} \mathrm{m}^{-1}\right)$ & $0 \%(0 / 11)$ & - & - & - & $0 \%(0 / 8)$ & - & $0 \%(0 / 3)$ & ND & ND \\
\hline
\end{tabular}

ND, Not determined.

* Data from Zhang et al. (1992).

exhibited the classical growth and biochemical profile of M. smegmatis (Wayne \& Kubica, 1986) (Table 3). Thus, conventional biochemical testing could not distinguish between these three taxa.

Eight of eight ( $100 \%)$ isolates of $M$. smegmatis group 2 and three of three $(100 \%)$ isolates of $M$. smegmatis group 3 tested degraded PAS to catechol, whereas none of the $13(0 \%)$ isolates of the $M$. smegmatis group 1 were positive for PAS degradation. As noted above, isolates of all three taxa tested exhibited typical $M$. smegmatis biochemical profiles. This was also true with regard to carbohydrate utilization as sole carbon sources (Table 3). All isolates of $M$. smegmatis groups
1, 2 and 3 tested utilized D-glucitol (D-sorbitol), i-myoinositol, D-mannitol and L-rhamnose. Likewise, only $11-50 \%$ of all isolates tested were capable of utilizing citrate as a sole carbon source. Utilization of $\mathbf{L}$ arabinose, D-galactose, D-trehalose and D-xylose differed somewhat between the three taxonomic groups. Twenty of $20(100 \%)$ isolates of M. smegmatis group 1 utilized L-arabinose and D-xylose, while 19 of $20(95 \%)$ and 20 of $20(100 \%)$ isolates of $M$. smegmatis group 2 and five of eight $(63 \%)$ isolates of $M$. smegmatis group 3 were able to utilize these two substrates. Ten of $13(77 \%)$ and seven of eight $(88 \%)$ isolates of $M$. smegmatis group 1 and $M$. smegmatis group 3, respectively, and four of ten ( $40 \%)$ and three 
Table 4. MICs by broth microdilution including $M C_{50}$ and $M I C_{90}$ of $M$. smegmatis groups 1,2 and 3

\begin{tabular}{|c|c|c|c|c|c|c|c|c|c|c|c|c|}
\hline \multirow[t]{2}{*}{ Drug } & \multicolumn{4}{|c|}{ Group 1} & \multicolumn{4}{|c|}{ Group 2} & \multicolumn{4}{|c|}{ Group 3} \\
\hline & No. tested & MIC range & $\mathrm{MIC}_{50}$ & $\mathbf{M I C}_{90}$ & No. tested & MIC range & $\mathrm{MIC}_{50}$ & MIC $_{90}$ & No. tested & MIC range & $\mathrm{MIC}_{50}$ & $\mathrm{MIC}_{90}$ \\
\hline Amikacin & 25 & $\leqslant 0.25-1$ & $\leqslant 0.5$ & $\leqslant 1$ & 28 & $\leqslant 0 \cdot 25-4$ & 0.5 & $\leqslant 1$ & 8 & $2-16$ & 2 & 16 \\
\hline Tobramycin & 34 & $\leqslant 0 \cdot 25-2$ & $\leqslant 0 \cdot 5$ & 2 & 28 & $\leqslant 0.5-8$ & 2 & 8 & 8 & $>16->32$ & $>32$ & $>32$ \\
\hline Doxycycline & 23 & $\leqslant 0 \cdot 25-1$ & $\leqslant 0 \cdot 25$ & 0.5 & 28 & $\leqslant 0 \cdot 25-1$ & $\leqslant 0.25$ & 0.5 & 8 & $0 \cdot 5-4$ & 1 & 4 \\
\hline Clarithromycin & 17 & $\leqslant 0.063->128$ & 4 & $>128$ & 17 & $\leqslant 0 \cdot 25->128$ & 32 & $>128$ & 8 & $8->64$ & $>16$ & 64 \\
\hline Imipenem & 24 & $\leqslant 0.5-4$ & $\leqslant 0.5$ & 2 & 28 & $\leqslant 0 \cdot 5-8$ & 2 & 8 & 8 & $2-8$ & 4 & 8 \\
\hline Cefoxitin & 22 & $\leqslant 2-128$ & 8 & 64 & 28 & $\leqslant 8->256$ & 64 & $>256$ & 8 & $16-128$ & 32 & 64 \\
\hline Cefmetazole & 12 & $\leqslant 1-32$ & 16 & 32 & 22 & $\leqslant 2->128$ & 32 & $>128$ & 8 & $16-32$ & 16 & 32 \\
\hline Ciprofloxacin & 25 & $\leqslant 0.063-1$ & 0.25 & 1 & 28 & $\leqslant 0.063-1$ & $0 \cdot 25$ & 0.5 & 8 & $0 \cdot 25-2$ & 1 & 2 \\
\hline Sulfamethoxazole & 23 & $\leqslant 1-4$ & $\leqslant 1$ & 4 & 28 & $\leqslant 1-8$ & $\leqslant 1$ & 8 & 8 & $\leqslant 1-4$ & $\leqslant 1$ & 4 \\
\hline
\end{tabular}

$\mathrm{MIC}_{50}$, MIC which inhibits $50 \%$ of strains tested.

$\mathrm{MIC}_{90}$, MIC which inhibits $90 \%$ of strains tested.
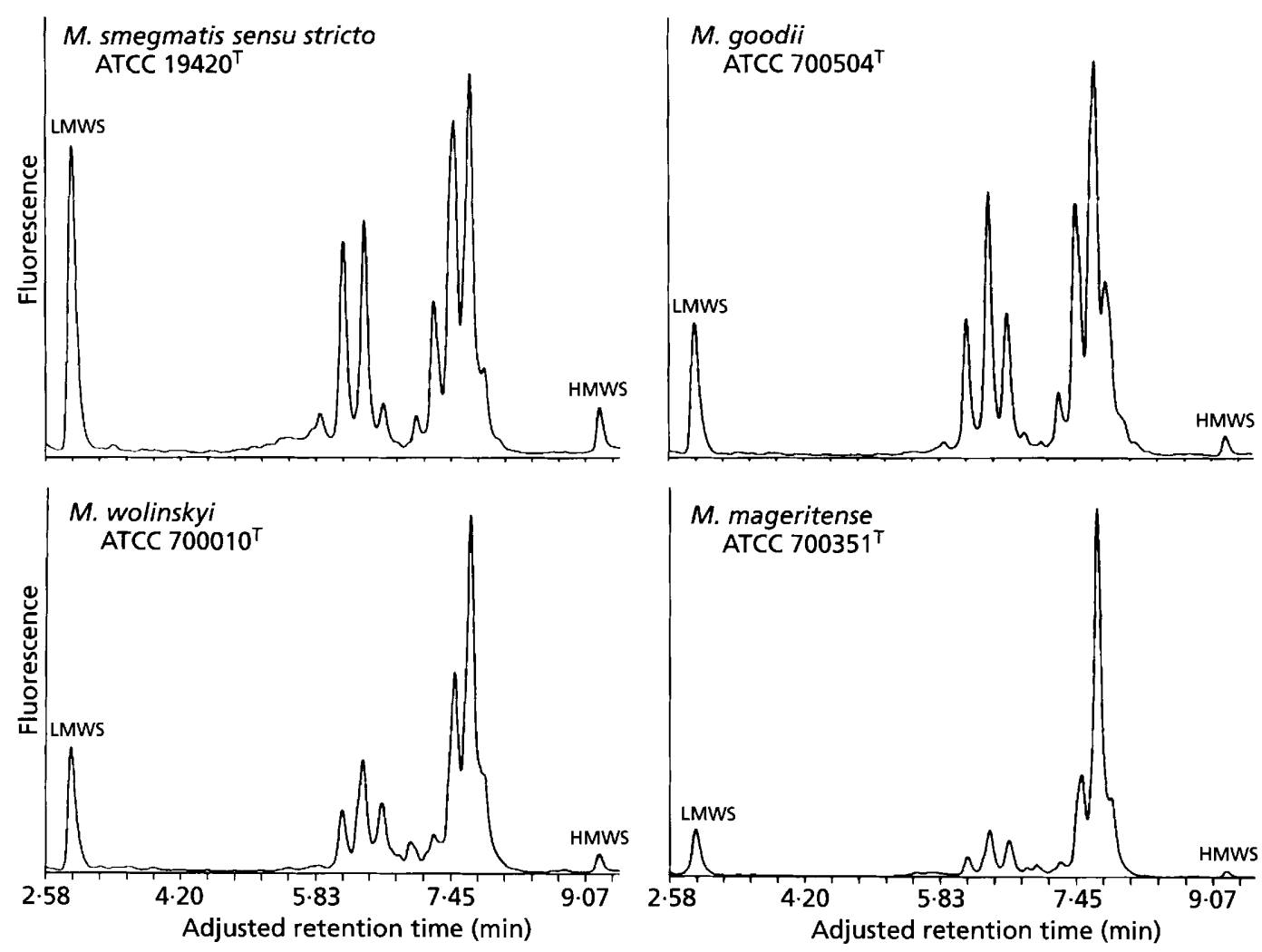

Fig. 1. Comparison of HPLC-generated mycolic acid 6,7-dimethoxy-4-coumarinylmethyl ester elution patterns for $M$. smegmatis group 1, $M$. mageritense and the proposed new species $M$. smegmatis groups 2 and 3. Abbreviations: LMWS, low molecular weight standard; and HMWS, high molecular weight standard.

of ten (30\%) isolates of $M$. smegmatis group 2, utilized D-galactose and D-trehalose, respectively, as sole carbon sources.

\section{Susceptibility testing}

Isolates of M. smegmatis group 1, M. smegmatis group 2 and $M$. smegmatis group 3 were susceptible to amikacin and sulfamethoxazole, intermediately sus- ceptible to ciprofloxacin and doxycycline, and variably susceptible to cefmetazole, cefoxitin and clarithromycin. Isolates of $M$. smegmatis group 3 exhibited higher MICs to amikacin, doxycycline and tobramycin, and lower MICs to cefoxitin than the other two species (Table 4). Isolates of all three taxa were susceptible to $5 \mu \mathrm{g}$ ethambutol ml $\mathrm{m}^{-1}$ and resistant to $25 \mu \mathrm{g}$ rifampin $\mathrm{ml}^{-1}$ and $10 \mu \mathrm{g}$ isoniazid $\mathrm{ml}^{-1}$ (Table 3 ). By comparison, $M$. mageritense was resistant to 


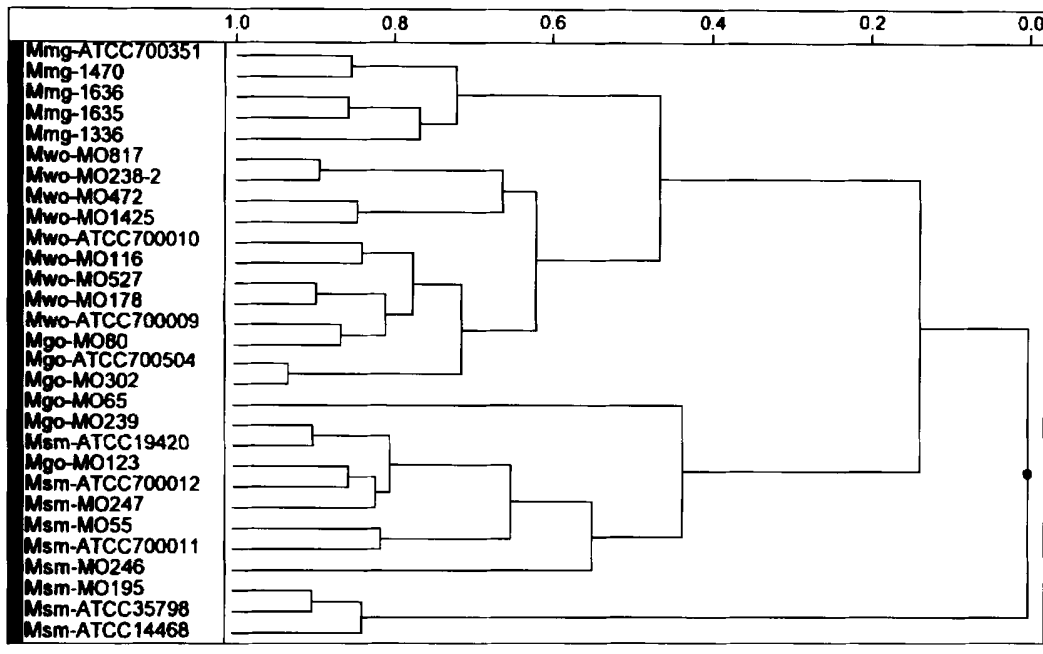

Fig. 2. Dendrogram illustrating phylogenetic relationships between $M$. smegmatis group $1, M$. mageritense and the proposed new species $M$. smegmatis groups 2 and 3 based on mycolic acid composition analyses by HPLC. Abbreviations: Msm, M. smegmatis group 1; Mgo, M. smegmatis group 2; Mwo, $M$. smegmatis group 3 ; and $\mathrm{Mmg}, M$. mageritense. Scale indicates the similarity index. ethambutol as well as isoniazid (Domenech et al., 1997).

Disk zone sizes were determined for $30 \mu \mathrm{g}$ chloramphenicol and $10 \mu \mathrm{g}$ tobramycin. Chloramphenicol zones were compared for 40 selected isolates of the three species. Twenty isolates of $M$. smegmatis group 1 , including all five reference strains, had zones of inhibition to the chloramphenicol disk $\geqslant 10 \mathrm{~mm}$ with a range of 11-44 mm and a mean zone diameter of 17 $\mathrm{mm}$. Twelve $M$. smegmatis group 2 isolates had zones of inhibition $\geqslant 10 \mathrm{~mm}$ with a range of $10-27 \mathrm{~mm}$ and a mean of $15 \mathrm{~mm}$. Conversely, all eight isolates of $M$. smegmatis group 3 exhibited zones of 6-12 $\mathrm{mm}$ with a mean of $9 \mathrm{~mm}$. Only four of the eight isolates had zones $>10 \mathrm{~mm}$.

Tobramycin disk diffusion zone diameters were determined for 52 isolates and, as noted (Wallace et al., 1988), were used for initial separation of the three taxonomic groups. Isolates of $M$. smegmatis group 1 were susceptible to tobramycin by disk diffusion, $M$. smegmatis group 1 isolates exhibited intermediate susceptibility to tobramycin, and $M$. smegmatis group 3 isolates were resistant to tobramycin (Table 3 ).

\section{HPLC}

FL-HPLC chromatograms aligned by using the two internal standards resulted in highly coincident peaks (Fig. 1). Strains analysed by both FL-HPLC and UVHPLC yielded highly comparable chromatograms (data not shown). M. smegmatis groups 1, 2, 3 and $M$. mageritense produced two cluster mycolic acid patterns; each cluster contained four to five peaks. These same four taxa differed in the relative abundance of both the front and rear clusters and the peaks within a cluster.

All strains of $M$. mageritense clustered strongly and apart from the other three taxa (Fig. 2). M. smegmatis group 3 strains yielded moderately conserved patterns that were intermediate between $M$. mageritense and some strains of $M$. smegmatis group 2. $M$. mageritense and $M$. smegmatis group 3 were characterized by low front cluster height; the height of the tallest front cluster peak ranged from 0.13 to 0.17 and 0.28 to 0.40 of the tallest rear cluster peak for $M$. mageritense and $M$. smegmatis group 3, respectively. M. mageritense and $M$. smegmatis group 3 strains produced a rear cluster with a single prominent peak at approximately 7.68 min ART; the second tallest rear cluster peak ranged from 0.27 to 037 and 0.46 to 0.69 of the tallest peak for $M$. mageritense and $M$. smegmatis group 3, respectively.

Compared to either $M$. mageritense or $M$. smegmatis group 3, M. smegmatis group 1 and $M$. smegmatis group 2 strains showed more diversity in their patterns and exhibited substantial front cluster peaks; the tallest front cluster peak was $\geqslant 0.50$ of the tallest rear cluster peak, strains MO195, ATCC 14468 and ATCC 35798 excepted. $M$. smegmatis group 1 was differentiated from $M$. smegmatis group 3 and $M$. mageritense by the relative abundance, within the rear cluster, of a peak at approximately $7.48 \mathrm{~min}$ ART. Most $M$. smegmatis group 1 and 2 strains were differentiated by the relative height of a peak at approximately $6.14 \mathrm{~min}$ ART to the tallest front cluster peak; this value was generally $\geqslant 0.81$ and $\leqslant 0.79$ for $M$. smegmatis groups 1 and 2 , respectively.

The HPLC patterns produced by $M$. smegmatis groups 1, 2, 3 and $M$. mageritense could be distinguished from the closely related patterns produced by the $M$. fortuitum group ( $M$. fortuitum, $M$. fortuitum third biovariant and $M$. peregrinum) based on an ART difference of a prominent peak in the front cluster. For $M$. smegmatis groups 1, 2, 3 and $M$. mageritense, this peak ranged from 6.38 to $6.43 \mathrm{~min}$ ART. For the $M$. fortuitum group, the corresponding peak ranged from 6.33 to 6.34 min ART.

Examples of the typical HPLC patterns for these three 


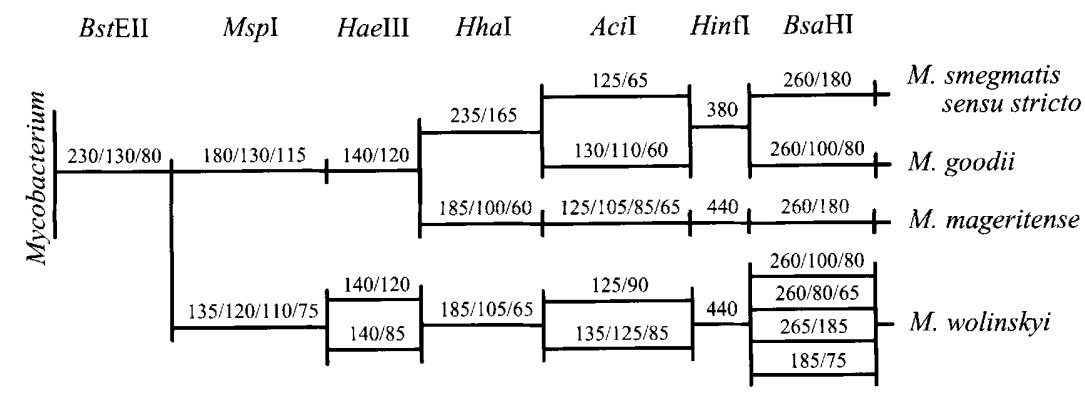

Fig. 3. Scheme for the differentiation of $M$. smegmatis group 1, $M$. mageritense and $M$. smegmatis groups 2 and 3 by PRA of a 439 bp segment of the $h s p-65$ gene.

$M$. smegmatis groups as well as $M$. mageritense are shown in Fig. 1. A dendrogram of the relatedness among these same taxa based on mycolic acid composition is shown in Fig. 2.

\section{GLC}

GLC-derived fatty acid profiles for isolates $M$. smegmatis groups 1, 2, 3 and $M$. mageritense did not differ from one another. Comparison of these profiles to fatty acid profiles for $M$. chelonae and $M$. fortuitum revealed some quantitative differences but there were no qualitative differences. No 2-methyl branchedchained fatty acids were observed in the fatty acid profiles for any of the rapidly growing mycobacterial isolates analysed in this study.

\section{$\mathbf{G}+\mathbf{C}$ composition of DNA}

The $\mathrm{G}+\mathrm{C}$ content of DNA for isolates of $M$. smegmatis group 3 was $68 \mathrm{~mol} \%$ for strain ATCC 700009 and $66 \mathrm{~mol} \%$ for ATCC strain $700010^{\mathrm{T}}$, respectively, with a mean value of $68 \pm 2 \mathrm{~mol} \%$ for all eight isolates of $M$. smegmatis group 3. The type strain ATCC $700504^{\mathrm{T}}$ of $M$. smegmatis group 2 had a G $+\mathrm{C}$ content of $66 \mathrm{~mol} \%$. The type strain ATCC $700351^{\mathrm{T}}$ of $M$. mageritense had a $\mathrm{G}+\mathrm{C}$ content of $66 \mathrm{~mol} \%$, and the mean value for all five isolates of $M$. mageritense was $67 \pm 3 \mathrm{~mol} \%$.

\section{PRA of the 439 bp hsp-65 gene sequence}

Sixty-one isolates were evaluated by PRA, including the five published strains of $M$. mageritense (Domenech et al., 1997) (including ATCC $700351^{\mathrm{T}}$ ), 25 isolates of $M$. smegmatis group 1 (including ATCC 14468, ATCC 35798, ATCC 700011, ATCC 700012 and ATCC $19420^{\mathrm{T}}$ ), $23 \mathrm{M}$. smegmatis group 2 isolates (including ATCC $700504^{\mathrm{T}}$ ), and eight $M$. smegmatis group 3 isolates (including ATCC 70009 and ATCC $700010^{\mathrm{T}}$ ). BstEII digests of PCR amplification products produced similar PRA patterns from all 61 $(100 \%$ ) isolates examined (Fig. 3). Digests with $M s p$ I readily differentiated isolates of $M$. smegmatis group 3 from those of $M$. smegmatis groups 1,2 and $M$. mageritense as shown in Figs 3 and 4a; lanes 12-14, 1

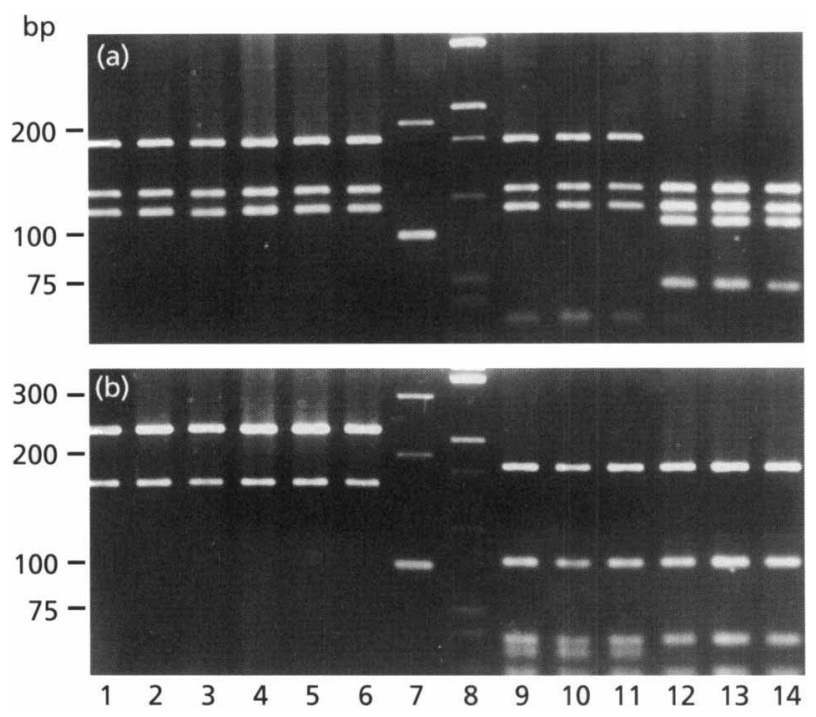

Fig. 4. (a) Mspl- and (b) Hhal-derived PRA band patterns for isolates of $M$. smegmatis group $1, M$. mageritense and $M$. smegmatis groups 2 and 3 . Lanes $1-3, M$. smegmatis group 1 isolates ATCC 19420', ATCC 700011 and ATCC 700012; lanes $4-6$, M. smegmatis group 2 isolates ATCC $700504^{\top}$, Mo 57 and Mo 65; lanes 7 and 8, sizes markers (100 bp and pGEM base pair ladders, respectively); lanes $9-11, M$. mageritense isolates 1636 , 1635 and 1336; and lanes 12-14, M. smegmatis group 3 isolates ATCC 700009, ATCC $700010^{\top}$ and Mo 116.

to 3, 4-6 and 9-11, respectively. HhaI-derived PRA patterns separated $M$. mageritense and $M$. smegmatis group 3 from $M$. smegmatis groups 1 and 2 (Figs 3 and 4b; lanes 9-11, 12-14, 1-3 and 4-6, respectively). PRA patterns with $A c i$ I further differentiated all four species (Figs 3 and 5a). The combination of MspI-, HhaI-, $A c i$ I- and $B s a \mathrm{HI}$-derived (Figs 3 and $5 \mathrm{~b}$ ) PRA patterns enabled complete differentiation of all four species.

Isolates of $M$. smegmatis groups 1 and 2 exhibited only a single PRA pattern with each enzyme. Three of the eight isolates of $M$. smegmatis group 3 exhibited a second PRA pattern with $\mathrm{HaeIII}$ and $A c i$ I that differed from the common pattern for the majority of isolates as shown in Fig. 3. Intraspecies polymorphism with $B s a \mathrm{HI}$ resulting in three different PRA patterns that differed from the common pattern for the majority of isolates was also observed for these isolates (Fig. 3). 
Mycobacterium wolinskyi sp. nov. and $M$. goodii sp. nov.
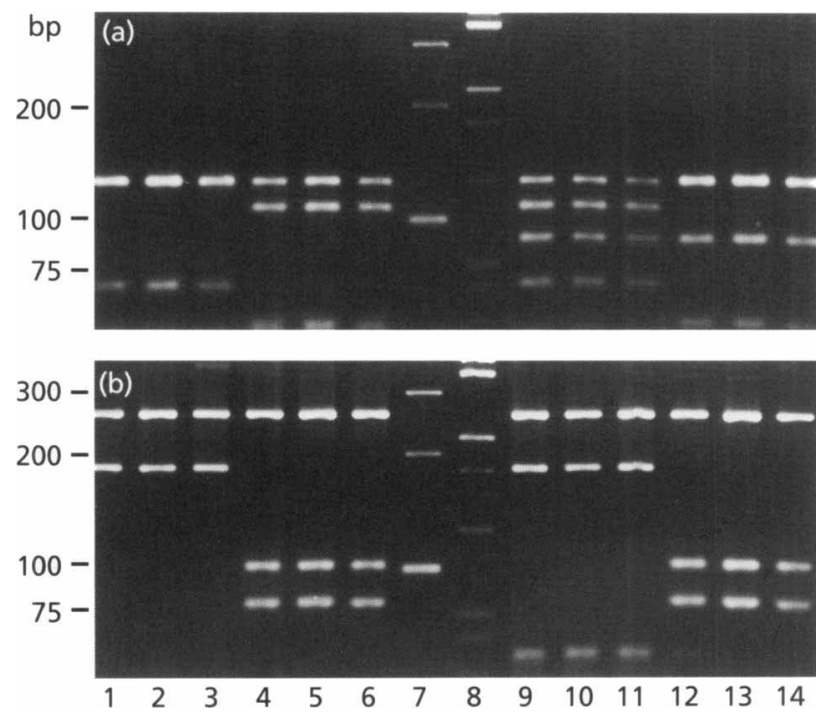

Fig. 5. (a) Acil- and (b) BsaHI-derived PRA band patterns for isolates of $M$. smegmatis group 1, $M$. mageritense and $M$. smegmatis groups 2 and 3. Lanes as for Fig. 4.

Four of $25(16 \%)$ clinical and reference isolates with a tobramycin susceptibility pattern of $M$. smegmatis group 1 subjected to PRA had a $M$. smegmatis group 2 PRA pattern (with the latter considered to be the species definitive test). All other isolates from the three tobramycin susceptibility groups matched the PRA grouping.

\section{5 rRNA sequencing}

$M$. smegmatis group 1 isolates ATCC 14468 and ATCC $19420^{\mathrm{T}}, M$. smegmatis group 2 isolate ATCC $700504^{\mathrm{T}}, M$. mageritense isolate ATCC $700351^{\mathrm{T}}$, and six M. smegmatis group 3 isolates (ATCC 700009, ATCC $700010^{\mathrm{T}}$, MO 178, MO 472, MO 527 and MO 116) were selected for $16 \mathrm{~S}$ rRNA sequence analysis of the hypervariable regions A and B (Kirschner et al., 1993b). By comparing the sequence of hypervariable region A, $M$. smegmatis groups 1,2 and 3 were readily differentiated from one another as well as from $M$. mageritense and members of the $M$. fortuitum complex (Table 5). In addition to other base pair changes, the $M$. smegmatis groups 1 and 2 isolates had an extra cytosine inserted at two sites in the hypervariable region A that corresponded to positions $182-183$ and 199-200 in the comparable region of Escherichia coli. With the exception of a small group of thermotolerant pigmented species, the former two species are the only rapidly growing mycobacterial species or taxa that have this extra cytosine. Similar insertions were not seen with any of the 6 isolates of $M$. smegmatis group 3 (Table 5).

Table 5. Signature nucleotides within the hypervariable region A of the 16S rRNA gene (the first nucleotide corresponds to $E$. coli position 175) for the pathogenic rapidly growing mycobacteria using $M$. tuberculosis as the reference species

Sequences obtained in part from references Domenech et al. (1997), Kirschner et al. (1992a, b), Springer et al. (1995). -, =- no

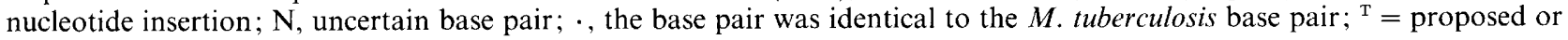
accepted type strain.

\begin{tabular}{|c|c|c|c|c|c|c|c|c|c|c|c|}
\hline \multirow{2}{*}{$\frac{\text { Taxon/species }}{M . \text { tuberculosis-M. bovis }}$} & \multicolumn{11}{|c|}{ Signature sequence* } \\
\hline & $\mathrm{CGG}$ & ATA & GG- & ACCA & CGG & GAT & GCA & TGT & $\mathrm{C}-\mathrm{TT}$ & GTG & GTG \\
\hline$M$. fortuitum ATCC $6841^{\mathrm{T}}$ & $\cdots \mathrm{A}$ & $\cdots$ & $\mathrm{T} \cdot-$ & $\cdots$ & $\cdots \mathrm{C}$ & $\cdot \mathrm{C}$. & $\mathrm{T} \cdots$ & $\cdots \mathrm{G}$ & TG.- & $\cdots$ & $\cdots$ \\
\hline$M$. peregrinum ATCC $14467^{\mathrm{T}}$ & $\cdots \mathrm{A}$ & $\ldots$ & $\mathrm{T} \cdot-$ & $\cdots G$ & $\cdots \mathrm{C}$ & $\mathrm{AC}$ & $\mathrm{T} \cdot \mathrm{C}$ & $\cdots \mathrm{G}$ & $\mathrm{T}-\mathrm{G}$ & $\cdots$ & $\cdots$ \\
\hline $\begin{array}{l}\text { M. fortuitum third biovariant complex } \\
\text { (sorbitol-positive) ATCC } 49403^{\mathrm{T}} \text {, } \\
\text { ATCC } 49934 \text { and ATCC } 49936\end{array}$ & $\cdots$ & $\cdots$ & $\cdots$ & $\cdots$ & $\cdots \mathrm{C}$ & $\cdot \mathrm{C}$. & $\mathrm{T} \cdot$ & $\cdots \mathrm{G}$ & $\mathrm{T}-\mathrm{G} \cdot$ & $\cdots$ & $\cdots$ \\
\hline $\begin{array}{l}\text { M. fortuitum third biovariant complex } \\
\text { (sorbitol-negative) ATCC } 49404^{\mathrm{T}} \text {, } \\
\text { ATCC } 49935 \text {, ATCC } 49937, \\
\text { ATCC } 49939\end{array}$ & $\cdots \mathrm{A}$ & $\cdots$ & $\cdots$ & $\cdots \mathrm{G}$ & $\cdots \mathrm{C}$ & $\mathrm{TC}$. & $\mathrm{T} \cdot$ & $\cdots G$ & G-G. & $\cdots$ & $\cdots$ \\
\hline M. abscessus АТCC $19977^{\mathrm{T}}$ & $\cdots$ & $\cdots$ & $\cdots$ & $\cdots$ & $\cdot \mathrm{AC}$ & $\mathrm{AC} \cdot$ & $\mathrm{T} \cdot$ & $\cdots \mathrm{G}$ & TG-A & $\cdots$ & $\cdots$ \\
\hline M. chelonae ATCC $35752^{\mathrm{T}}$ & $\cdots$ & $\cdots$ & $\cdots$ & $\cdots$ & $\cdot \mathrm{AC}$ & $\mathrm{AC}$ & $\mathrm{T} \cdot$ & $\cdots \mathrm{G}$ & TG-A & $\cdots$ & $\cdots$ \\
\hline $\begin{array}{l}\text { M. mucogenicum ATCC } 49649, \text { ATCC } \\
49650^{\mathrm{T}} \text {, АТCC } 49651\end{array}$ & $\cdots \mathrm{A}$ & $\cdots$ & $\cdots$ & $\cdots$ & $\cdots \mathrm{C}$ & $\cdot \mathrm{C} \cdot$ & $\mathrm{T} \cdot$ & $\cdots G$ & TG-• & $\cdots$ & $\cdots$ \\
\hline $\begin{array}{l}\text { M. smegmatis group } 1 \text { ATCC } 19420^{\mathrm{T}} \text {, } \\
\text { ATCC } 14468\end{array}$ & $\cdots \mathrm{A}$ & $\cdots$ & $\mathrm{CAC}$ & $\mathrm{C} \cdot \mathrm{TG}$ & $\cdot \mathrm{T} \cdot$ & $\cdot \mathrm{TC}$ & $\cdots$ & $\cdots \mathrm{G}$ & $\cdot$ CTG & $\cdots \mathrm{A}$ & $\cdot \mathrm{G}$ \\
\hline M. smegmatis group 2 ATCC $700504^{\mathrm{T}}$ & $\cdots \mathrm{A}$ & $\cdots$ & $\mathrm{TAC}$ & $\mathrm{C} \cdot \mathrm{TG}$ & $\cdot \mathrm{T} \cdot$ & $\cdot \mathrm{TC}$ & $\cdots$ & $\cdots \mathrm{G}$ &. CTG & $\cdots$ & $\cdot \mathrm{G}$ \\
\hline $\begin{array}{l}\text { M. smegmatis group } 3 \text { ATCC } 700010^{\mathrm{T}} \text {, } \\
\text { MO 178, MO 472, MO 527, MO } 116 \# 2\end{array}$ & $\cdots$ & $\cdots$ & $\cdots$ & $\cdots$ & $\cdots$ & TC. & $\cdots$ & $\cdots \mathrm{G}$ & $A \cdot \cdot$ & $\cdots$ & $\cdots$ \\
\hline ATCC 700009 (MF 238) & $\cdots \mathrm{A}$ & $\cdots$ & $\cdots$ & $\cdots \mathrm{TC}$ & $\cdots$ & $\mathrm{NC} \cdot$ & $\cdots$ & $\cdots \mathrm{G}$ & TN-. & $\cdots$ & $\cdots$ \\
\hline M. mageritense ATCC $700351^{\mathrm{T}}$ & $\cdots \mathrm{A}$ & $\cdots$ & $\cdots$ & $\cdots \mathrm{TC}$ & $\cdots$ & $\mathrm{AC}$ & $\cdots$ & $\cdots \mathrm{G}$ & TC-. & $\cdot \mathrm{G} \cdot$ & $\cdots$ \\
\hline
\end{tabular}




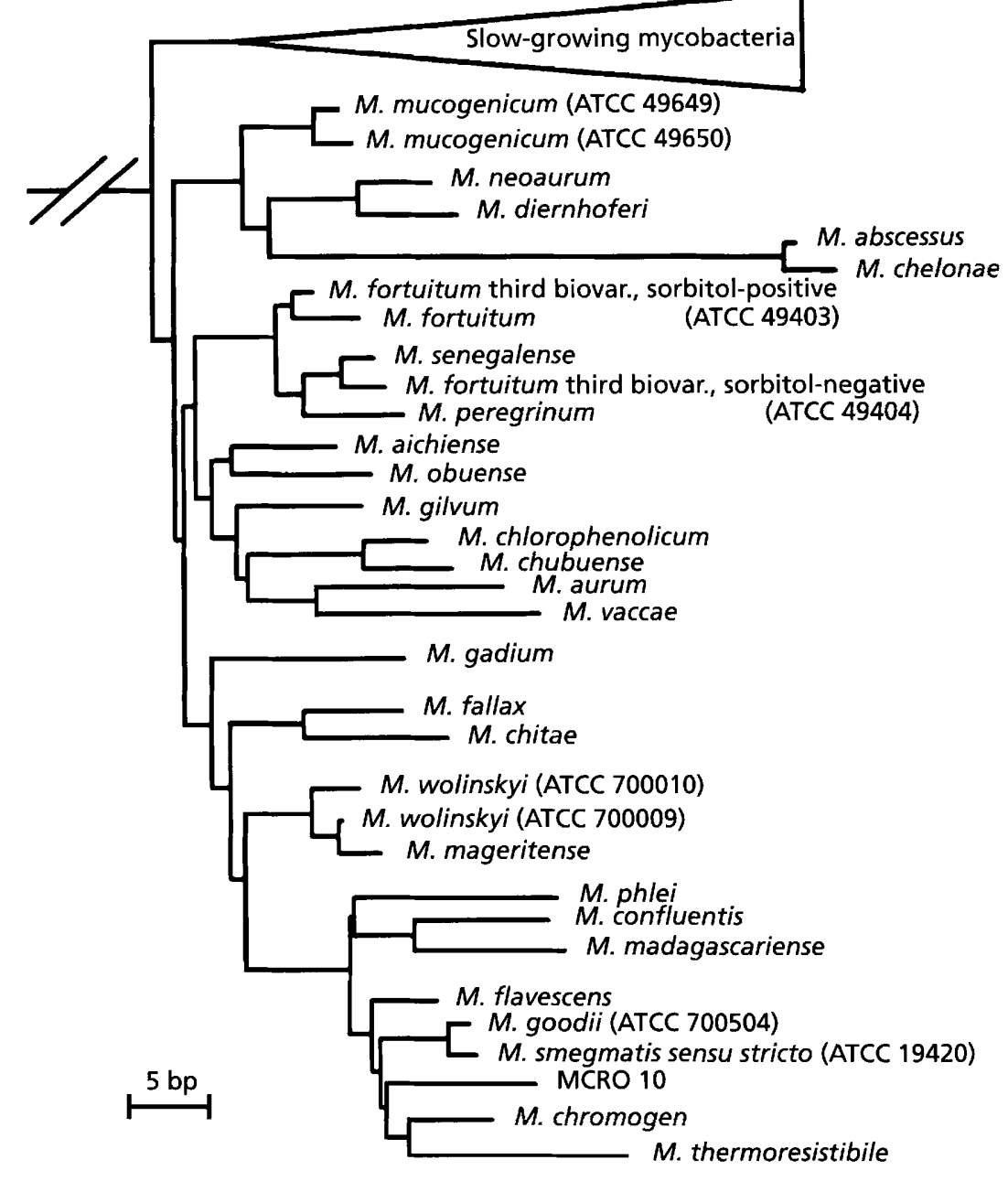

Fig. 6. Dendrogram illustrating the phylogenetic relationships among 30 species and taxa of rapidly growing mycobacteria including $M$. smegmatis group 1 ( $M$. smegmatis sensu stricto) (ATCC $19420^{\top}$ ), $M$. mageritense (938 ${ }^{\top}$ [Domenech et al., 1997]), $M$. smegmatis group 2 ( $M$. goodii) (ATCC $700504)$ and $M$. smegmatis group $3(M$. wolinskyi) (ATCC 700009 and ATCC $700010^{\top}$ ).
Isolates were then selected from each of these three groups and these isolates were subjected to complete 16S rRNA sequencing, namely $M$. smegmatis group 1 (ATCC $19420^{\mathrm{T}}$ ), M. smegmatis group 2 (ATCC $700504^{\mathrm{T}}$ ), $M$. mageritense 1336 and $M$. smegmatis group 3 (ATCC 700009 and ATCC $700010^{\mathrm{T}}$ ). The sequence of $M$. smegmatis group 1 ATCC $19420^{\mathrm{T}}$ was identical to the EMBL sequence for $M$. smegmatis group 1 ATCC 14468.

A dendrogram illustrating the 16S rRNA phylogenetic relationships among species of the rapidly growing mycobacteria including $M$. smegmatis groups $1,2,3$ and $M$. mageritense is shown in Fig. 6, while a summary of the degree of relatedness based on Hamming distances is given in Table 6. M. smegmatis groups 1 and 2 showed a close phylogenetic relationship with a Hamming distance of 4 . These two species share a common branch point in the 16S rRNA phylogenetic tree (Fig. 6) and share the characteristic insertion of two cytosine nucleotides in the hypervariable region $\mathrm{A}$ as noted above in Table $6 . M$. smegmatis groups 1 and 2 were clustered with other thermotolerant species (e.g. M. phlei, Mycobacterium thermoresistibile, etc.) with this same cytosine insertion.

The two isolates of $M$. smegmatis group 3 (ATCC 700009 and ATCC $700010^{\mathrm{T}}$ ) exhibited sequences that differed from those of all previously described species of rapidly growing mycobacteria. They shared a common, but clearly separated, branch point in the $16 \mathrm{~S}$ rRNA phylogenetic tree that was also shared with $M$. mageritense as illustrated in Fig. 6 . Five of the six $M$. smegmatis group 3 isolates including the type strain ATCC $700010^{\mathrm{T}}$ exhibited identical $16 \mathrm{~S}$ rRNA sequences that differed from that of $M$. smegmatis group 1 by a Hamming distance of 18 , from $M$. smegmatis group 2 by a distance of 16 , from $M$. mageritense by a distance of 9 , and from 28 other species and taxa of rapidly growing mycobacteria by a distance of 16 or greater (Table 6). The hypervariable region A rRNA sequence of the sixth $M$. smegmatis group 3 isolate (ATCC 700009) differed from those of the other five isolates by $4 \mathrm{bp}$. Unlike the hypervariable region A sequences that were different for each species, the hypervariable region B sequences for $M$. mageritense, $M$. smegmatis groups 2 and 3 were 


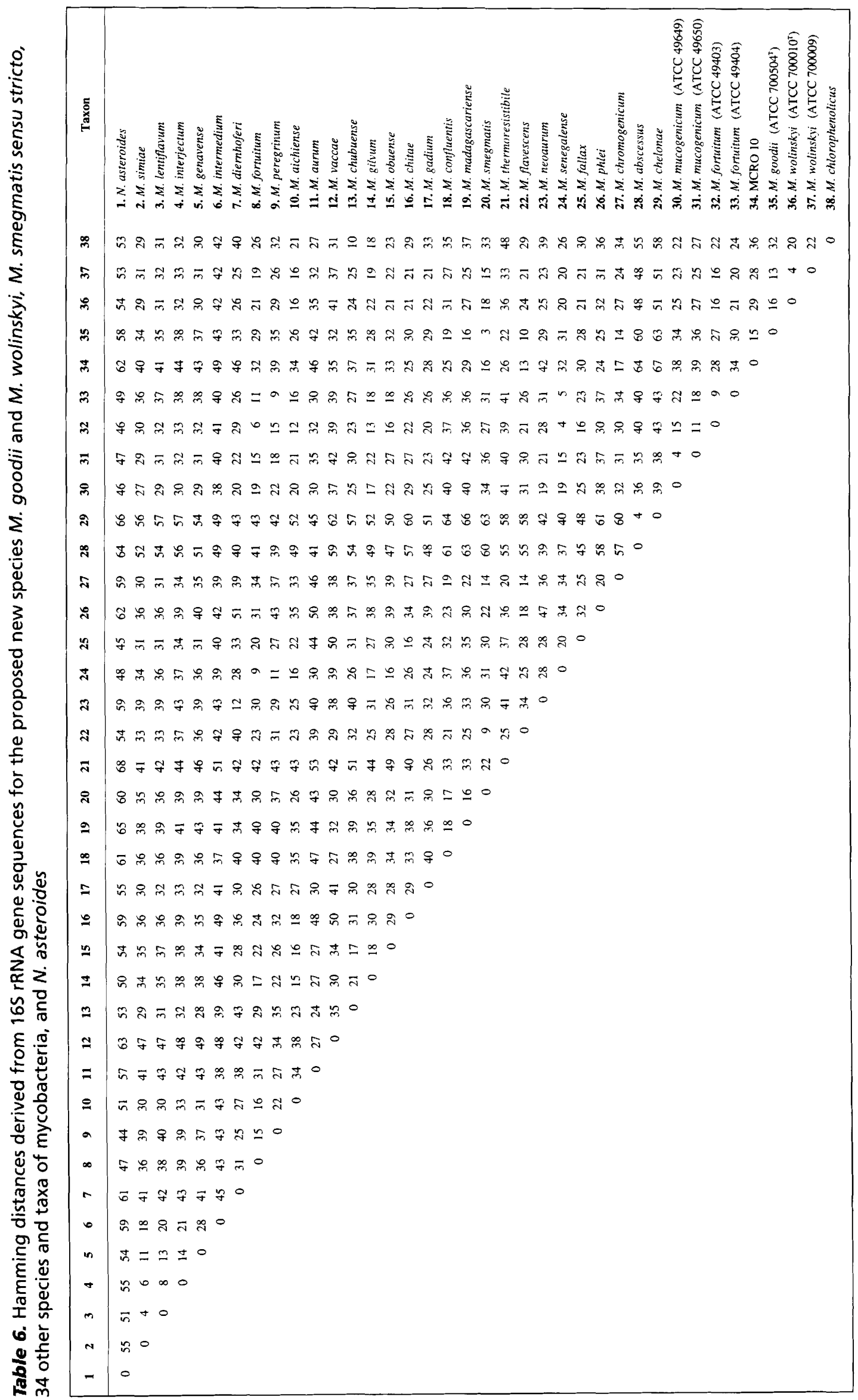


Table 7. Second region in the 165 rRNA gene (corresponding to $E$. coli positions 1001-1027) that differentiated the common species belonging to the $M$. fortuitum complex, and $M$. smegmatis groups 1 and 3

., The base pair was identical to the $M$. tuberculosis base pair; ${ }^{\mathrm{T}}$, proposed or accepted type strain. Strain numbers are the same as in Table 4.

\begin{tabular}{|c|c|}
\hline Taxon & Sequence \\
\hline M. tuberculosis - M. bovis & ACAGGACGCGTCTAGAGATAGGCGTT \\
\hline M. fortuitum & ......AC.GC.....GT.GT. \\
\hline$M$. peregrinum & $\ldots \ldots \ldots$ CAG. .....TTG. \\
\hline$M$. fortuitum third biovariant complex (sorbitol-negative) & $\ldots \ldots \ldots$ CAG . . . . . TTG . \\
\hline M. abscessus & $\ldots \ldots \ldots$ TAC........ \\
\hline M. chelonae & G. . . TA. . . \\
\hline M. fortuitum third biovariant complex (sorbitol-positive) & $\ldots \ldots \ldots$ CGGC $\ldots \ldots$ GTCG $\ldots$ \\
\hline M. mucogenicum & $\ldots \ldots \ldots$ CGGC $\ldots \ldots$ GTCG $\ldots$ \\
\hline M. mageritense ATCC $700351^{\mathrm{T}}$ & $\ldots \ldots \ldots$ CGGC $\ldots \ldots$ GTCG $\ldots$ \\
\hline M. smegmatis group 1 & $\ldots \ldots \ldots$ CGGC $\ldots \ldots$ GTCG $\ldots$ \\
\hline M. smegmatis group 2 (ATCC $700504^{\mathrm{T}}$ ) & $\ldots \ldots \ldots$ CGGC $\ldots \ldots$ GTCG $\ldots$ \\
\hline \multicolumn{2}{|l|}{ M. smegmatis group 3} \\
\hline ATCC $700010^{\mathrm{T}}$ (MO739), MO 178, MO 472, MO 527, & $\ldots \ldots \ldots$ TCGGC $\ldots \ldots$ GTCG $\ldots$ \\
\hline \multicolumn{2}{|l|}{ MO $116 \# 2$} \\
\hline ATCC 700009, (MF 238) & $\ldots \ldots \ldots$ TCGGC......GTCG $\ldots$ \\
\hline
\end{tabular}

Table 8. Levels of DNA-DNA homology (\%) between $M$. smegmatis groups 2 and 3 and related taxa of rapidly growing mycobacteria

\begin{tabular}{|c|c|c|c|c|c|}
\hline Species/strain & $\begin{array}{l}\text { M. mageritense } \\
\text { ATCC } 700351^{\mathrm{T}}\end{array}$ & $\begin{array}{c}\text { Group 1 } \\
{\text { ATCC } 19420^{\mathrm{T}}}^{\text {a }}\end{array}$ & $\begin{array}{c}\text { Group } 2 \\
\text { ATCC } 700504\end{array}$ & $\begin{array}{c}\text { Group 3 } \\
\text { ATCC } 700009\end{array}$ & $\begin{array}{c}\text { Group 3 } \\
\text { ATCC } 700010^{\mathrm{T}}\end{array}$ \\
\hline \multicolumn{6}{|l|}{ M. mageritense } \\
\hline ATCC $700351^{\mathrm{T}}$ & 100 & - & - & - & - \\
\hline \multicolumn{6}{|l|}{ M. smegmatis group 1} \\
\hline ATCC $19420^{T}$ & $4 \pm 3$ & 100 & - & - & _- \\
\hline \multicolumn{6}{|l|}{ M. smegmatis group 2} \\
\hline ATCC $700504^{\mathrm{T}}$ & $11 \pm 5$ & $39 \pm 14$ & 100 & - & - \\
\hline \multicolumn{6}{|l|}{ M. smegmatis group 3} \\
\hline ATCC 700009 & $13 \pm 4$ & $17 \pm 7$ & $17 \cdot 3 \pm 8$ & 100 & - \\
\hline ATCC $700010^{\mathrm{T}}$ & $11 \pm 6$ & $19 \pm 8$ & $21 \pm 8$ & $84 \pm 15$ & 100 \\
\hline MF1425 & $12 \cdot 2 \pm 1 \cdot 5$ & $14 \cdot 3 \pm 7$ & $19 \pm 5 \cdot 4$ & $99 \pm 7$ & $91 \pm 15$ \\
\hline MO116 & $11 \cdot 2 \pm 5$ & $11 \pm 4 \cdot 5$ & $19 \pm 5$ & $>100$ & $98 \pm 20$ \\
\hline MO178 & $7.7 \pm 1.5$ & $15 \pm 7$ & $16 \cdot 4 \pm 5 \cdot 6$ & $>100$ & $>100$ \\
\hline MO472 & $11 \cdot 2 \pm 3$ & $26 \pm 10$ & $23 \pm 9$ & - & $104 \pm 20$ \\
\hline MO527 & $9 \cdot 7 \pm 2 \cdot 7$ & $20 \pm 8$ & $19 \pm 8$ & - & $80 \pm 15$ \\
\hline MO817 & $5 \pm 0.5$ & $9 \pm 3$ & $18 \cdot 2 \pm 5$ & - & $65 \pm 7$ \\
\hline \multicolumn{6}{|l|}{ M. fortuitum } \\
\hline ATCC $6841^{\mathrm{T}}$ & $20 \cdot 2 \pm 5$ & $14 \pm 6$ & $17 \cdot 6 \pm 7$ & $23 \cdot 5 \pm 0.6$ & $13 \pm 3$ \\
\hline M. phlei IMRU 500 & $5 \cdot 7 \pm 8$ & $21 \pm 5$ & $17 \pm 8$ & $9 \pm 3$ & $11 \pm 1 \cdot 5$ \\
\hline
\end{tabular}

identical with $M$. smegmatis group 1. Separation of $M$. smegmatis group 3 from all other taxa could also be made by examining the $1001-1027$ bp region (i.e. signature sequences designated by the $E$. coli numbering system) depicted in Table 7 .

\section{DNA-DNA hybridization}

The mean results of four DNA-DNA hybridization analyses per sample are shown in Table 8. Most isolates of $M$. smegmatis group 3 showed $20 \%$ or less 
Mycobacterium wolinskyi sp. nov. and $M$. goodii sp. nov.
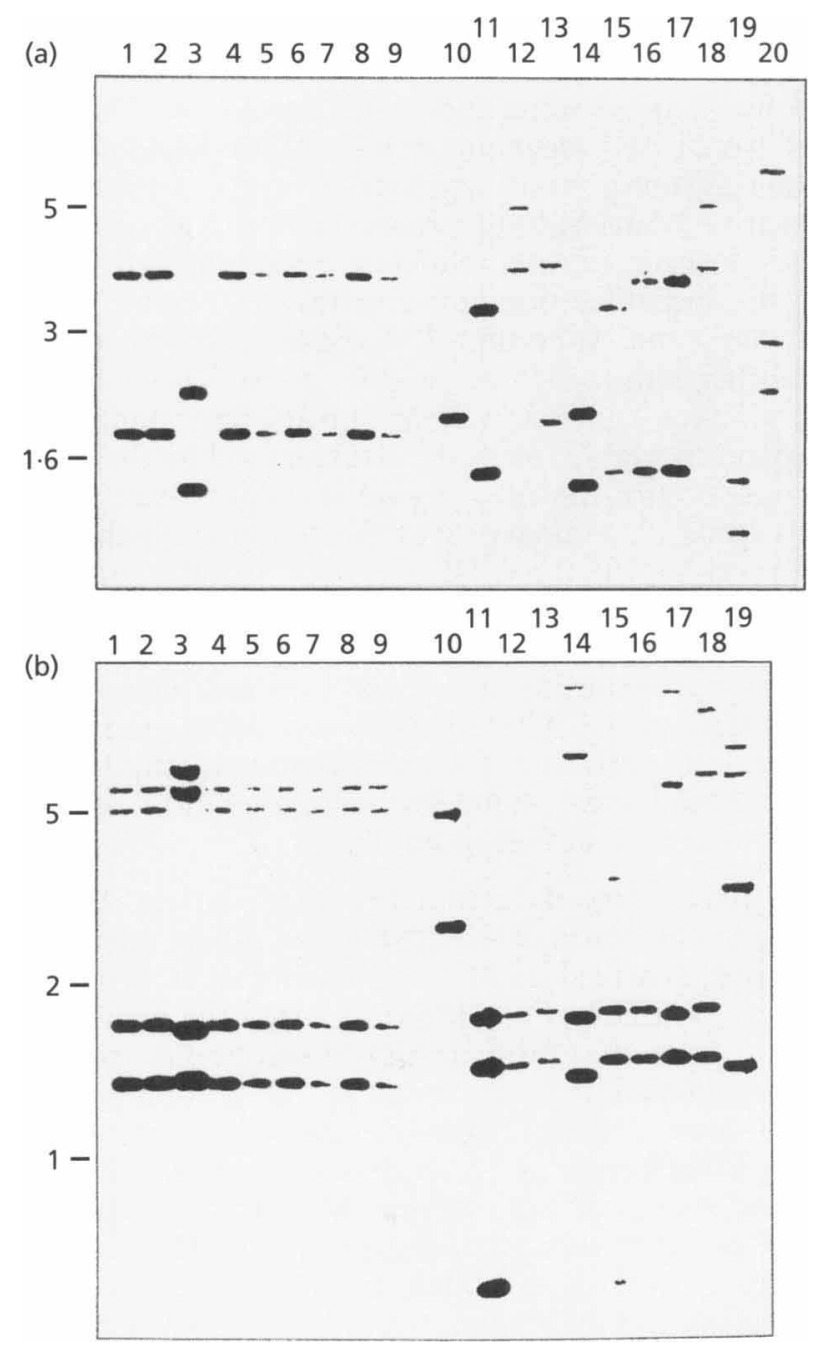

Fig. 7. BamHI- and Pstl-derived RFLP patterns of 165 rRNA genes from $M$. smegmatis groups 2 and 3 compared to $M$. mageritense, $M$. smegmatis and other species of rapidly growing mycobacteria, and $N$. asteroides. (a) BamHI digests. Lane $3, M$. goodii isolate ATCC $700504^{\top}$. Lanes 1,2 and 4-9, $M$. smegmatis group 3 isolates ATCC 700009, MO 1425, MO 116, MO 178, MO 472, MO 527, ATCC 700010 ${ }^{\top}$, MF 817, respectively. Lanes $10-20, M$. chelonae ATCC $35752^{\top}, M$. fortuitum ATCC $6841^{\top}, M$. fortuitum third biovar strain ATCC $49403^{\top}, M$. fortuitum third biovar strain ATCC 49404 ${ }^{\top}, M$. mageritense ATCC $700351^{\top}, M$. parafortuitum strain CNMIVS, $M$. peregrinum ATCC $14467^{\top}, M$. porcinum ATCC $27406^{\top}, M$. senegalense NCTC $10956^{\top}$, Mycobacterium sp., and $N$. asteroides strain CMNIVS. (b) Pstl digests. Lanes 1-19 are the same as for (a). Abbreviations: CIPT, Collection Institut Pasteur Unité de Tuberculose; NCTC, National Collection of Type Cultures, and CNMIVS, Centro Nacional de Microbiologia, Immunologia y Virologia Sanitarias.

homology with the type strains of $M$. mageritense (ATCC $700351^{\mathrm{T}}$ ) (Domenech et al., 1997), $M$. smegmatis group 1 (ATCC $19420^{\mathrm{T}}$ ) and $M$. smegmatis group 2 (ATCC $700504^{\mathrm{T}}$ ). Most isolates of $M$. smegmatis group 3 exhibited $90 \%$ or greater homology within the group including the two reference strains ATCC 700009 and ATCC $700010^{\mathrm{T}}$. The $M$. smegmatis group 2 strain ATCC $700504^{\mathrm{T}}$ exhibited only $39 \%$ homology with the $M$. smegmatis group 1 strain ATCC $19420^{\mathrm{T}}$.

\section{RFLP analysis of 16S rRNA gene}

Strain comparisons included $M$. abscessus, $M$. chelonae, M. fortuitum, M. fortuitum third biovariant sorbitol-positive, $M$. fortuitum third biovariant sorbitol-negative, $M$. mageritense, $M$. parafortuitum, $M$. peregrinum, $N$. asteroides, Mycobacterium porcinum, Mycobacterium senegalense, and $M$. smegmatis groups 1, 2 and 3.

The eight isolates of $M$. smegmatis group 3 gave identical RFLP hybridization patterns with two Bam HI-derived bands and four $P s t \mathrm{I}$-derived bands. These patterns were unique from all control strains. Likewise, isolates of $M$. smegmatis group 2 including the type strain ATCC $700504^{\mathrm{T}}$ gave a very different RFLP-hybridization pattern from that of the $M$. smegmatis group 3 isolates, and was unique from those of all other control strains (Fig. 7).

\section{DISCUSSION}

These three taxonomic groups exhibited typical characteristics of the genus mycobacterium. They grew well aerobically on Middlebrook $7 \mathrm{H} 10$ agar and Löwenstein-Jensen agar, were Gram-positive and appeared as acid-fast bacilli after staining with ZiehlNeelsen strain, contained mycobacterial typical mycolic acids and whole-cell fatty acids on HPLC and GLC, respectively, and exhibited more than $95 \%$ identity with other mycobacterial 16S rRNA sequences. Their mean of $67 \mathrm{~mol} \% \mathrm{G}+\mathrm{C}$ content is within the range of $62-70 \mathrm{~mol} \%$ typical of the genus mycobacterium (Wayne \& Kubica, 1986).

The genetic studies clearly delineated differences between the three $M$. smegmatis groups, including by PRA of the $65 \mathrm{kDa}$ heat-shock protein gene, $16 \mathrm{~S}$ rRNA RFLP patterns, 16S rRNA sequences and DNA-DNA pairing experiments. PRA with BstEII resulted in a single band pattern for $M$. mageritense, and $M$. smegmatis groups 1,2 and 3, including the ATCC reference strains, suggesting a close relationship between the groups. As previously demonstrated (Steingrube et al., 1995b; Telenti et al., 1993) the use of both BstEII and HaeIII separated these four taxa from all other rapidly growing mycobacterial species and taxa studied. The use of other restriction enzymes separated all of the four groups. Isolates of $M$. smegmatis groups 1 and 2 were differentiated from one another by two of the five restriction enzymes (AciI and $B s a \mathrm{HI}$ ) and from $M$. smegmatis group 3 by three enzymes ( $M s p \mathrm{I}$, HhaI and $A c i \mathrm{I})$.

By $16 \mathrm{~S}$ rRNA sequencing data, the common sequence seen in five of six $M$. smegmatis group 3 isolates differed from all other mycobacterial taxa, except $M$. mageritense, by a Hamming distance of at least 16. The Hamming distance of 9 between the common sequence for $M$. smegmatis group 3 (including ATCC $700010^{\mathrm{T}}$ ) 
and $M$. mageritense suggested these two species were more closely related to one another than to $M$. smegmatis groups 1 and 2. Likewise, the latter two taxa differed by a Hamming distance of 3 and thus are among the most closely related of all the mycobacterial taxa studied by $16 \mathrm{~S}$ rRNA sequencing. [A few mycobacterial species have fewer differences, e.g. Mycobacterium ulcerans and Mycobacterium marinum which differ by 2 bp (Tønjum et al., 1998) and Mycobacterium gastri and Mycobacterium kansasii which have identical 16S rRNA sequences (Rogall et al., 1990).]

M. smegmatis groups 2 and 3 exhibited $<40 \%$ DNA-DNA homology with one another or with $M$. mageritense or $M$. smegmatis group 1. Despite differing by a small Hamming distance of 4 in their $16 \mathrm{~S}$ rRNA sequences, $M$. smegmatis groups 1 and 2 exhibited different PRA patterns, phenotypic patterns, and $<40 \%$ DNA-DNA homology. This degree of relatedness is well less than the approximately $70 \%$ DNA-DNA relatedness recommended by Wayne et al. (1987). Conversely, M. smegmatis group 3 strains ATCC 700009 and ATCC $700010^{T}$ exhibited a Hamming distance of 4 , but were identical by PRA and phenotypic characteristics and exhibited $84 \%$ DNA-DNA homology.

FL-HPLC has been shown to be a sensitive and practical method for the identification of mycobacteria (Jost et al., 1995). In the current study, the relationships between the four species as determined by HPLC mycolic acid patterns were similar to those observed by 16S rRNA sequencing. Previously, Butler \& Kilburn (1990) reported that $M$. smegmatis could not be distinguished from the $M$. fortuitum group based on their HPLC patterns. However, in the current study a small but consistent difference in peak ART values clearly differentiated $M$. smegmatis groups 1,2 , 3 and $M$. mageritense from the $M$. fortuitum group.

Consistent with previous reports (Baba et al., 1988; Yassin et al., 1993), whole-cell fatty acid profiles were very homogeneous among the rapidly growing mycobacteria tested and did not distinguish between $M$. smegmatis groups 1, 2, 3, or $M$. mageritense. Apart from minor quantitative differences, fatty acid profiles also did not differentiate the latter species from $M$. chelonae or $M$. fortuitum. Valero-Guillén \& MartinLuengo (1983) reported that 2-methyl branched-chain fatty acids were detected only in isolates of $M$. fortuitum and M. smegmatis. These authors identified 2-methyl-2-octadecanoic acid in some but not all isolates of $\boldsymbol{M}$. fortuitum and have identified three different whole-cell fatty acid patterns in this species by GLC (Valero-Guillén \& Martin-Luengo, 1983). They have also reported 2,4-dimethyl-2-eicosenoic acid to be present in two of three isolates of $M$. smegmatis. Notably, the ATCC 607 strain of $M$. smegmatis lacked these acids. However, in the current study no 2-methyl branched-chain fatty acids were detected in any of the rapidly growing mycobacterial isolates tested, findings that parallel those of Yassin et al. (1993).
The phenotypic similarity of the three $M$. smegmatis groups is one of the major reasons $M$. smegmatis groups 2 and 3 went undetected until now. The three groups of $M$. smegmatis exhibited the same conventional growth and biochemical tests, including a negative $3 \mathrm{~d}$ arylsulfatase reaction, positive iron uptake and positive nitrate reductase reactions, growth at $45^{\circ} \mathrm{C}$, and utilization of D-glucitol (D-sorbitol), i-myoinositol and D-mannitol as sole carbon sources. Another reason $M$. smegmatis group 3 isolates have gone unrecognized was an insufficient number of isolates available for study, as evidenced by their $11 \%$ proportion of the $M$. smegmatis isolates examined in this study. A comparison of the common biochemical markers for the three $M$. smegmatis groups and other closely related rapidly growing mycobacteria is presented in Table 8. Routine laboratory testing of rapidly growing mycobacteria does not include PAS degradation, $68^{\circ} \mathrm{C}$ stable catalase assay, IEF of $\beta$ lactamases, PRA or DNA sequencing, methodologies that would have made earlier recognition of these proposed new species more likely.

The phenotypic data that provided the earliest and clearest indication of the existence of other taxa among isolates identified as M. smegmatis was susceptibility testing. Wallace et al. (1988) reported the presence of three different antimicrobial susceptibility patterns among clinical isolates. Based on the groupings in the 1988 study, these three groups of isolates, were designated herein as $M$. smegmatis groups 1, 2 and 3, respectively. All eight of the $M$. smegmatis group 3 isolates were resistant to tobramycin, the $M$. smegmatis group 2 isolates exhibited an intermediate level of resistance to tobramycin, while $M$. smegmatis group 1 isolates were susceptible.

\section{Description of Mycobacterium wolinskyi sp. nov.}

Mycobacterium wolinskyi (wo.lins'ky.i. M.L. gen. n. wolinskyi of Wolinsky, named after Emanuel Wolinsky in honour of, and in recognition for, significant contributions to the study of the non-tuberculous mycobacteria).

A Gram-positive, acid- and alcohol-fast bacillus that produces visible growth in $2-4 \mathrm{~d}$ as smooth to mucoid, off-white- to cream-coloured colonies on Middlebrook $7 \mathrm{H} 10$ and trypticase soy agar. No pigment is produced and growth occurs at 30,35 and $45^{\circ} \mathrm{C}$. Isolates grow on MacConkey agar without crystal violet and in the presence of $5 \% \mathrm{NaCl}$, are negative for arylsulfatase activity at $3 \mathrm{~d}$, and are positive for iron uptake and nitrate reductase. They produce low-level semi-quantitative catalase activity that is stable at $68^{\circ} \mathrm{C}, \mathrm{pH} 7 \cdot 0$. As sole carbon sources, $100 \%$ of isolates utilize Dglucitol (D-sorbitol), i-myo-inositol, D-mannitol and Lrhamnose, $88 \%$ utilize D-galactose and D-trehalose, and $50-63 \%$ utilize L-arabinose, citrate and D-xylose. The type strain is positive for galactose, arabinose, citrate, trehalose and xylose. Isolates are susceptible to amikacin and sulfamethoxazole, intermediately sus- 
Mycobacterium wolinskyi sp. nov. and $M$. goodii sp. nov.

ceptible to doxycycline and ciprofloxacin, variably susceptible to cefmetazole, cefoxitin, chloramphenicol and clarithromycin, and resistant to isoniazid, rifampin and tobramycin. Mycolic acids and wholecell fatty acids are typical of mycobacterial species. By PRA, isolates exhibit a unique, distinctive set of band patterns with $M s p I$ I $(135,120,110$ and 75 bp bands), HhaI (185, 105 and 65 bp bands) and Acil (125 and $90 \mathrm{bp}$ major band pattern, and 135,125 and $85 \mathrm{bp}$ minor band pattern). The proposed type strain, ATCC $700010^{\mathrm{T}}$ (MO739), was recovered from a post-surgical facial abscess in Switzerland and was originally identified as $M$. smegmatis (Pennekamp et al., 1997). An additional reference strain, ATCC 700009 (MO238), was isolated from a sternal wound infection following open heart surgery in Texas.

\section{Description of Mycobacterium goodii sp. nov.}

Mycobacterium goodii [good.i.i. M.L. gen. n. goodii of Good, named after Robert (Bob) Good, in honour of, and in recognition for, significant contributions to the study of mycobacteria].

A Gram-positive, acid- and alcohol-fast bacillus that produces visible growth in 2-4 d as smooth to mucoid, off-white- to cream-coloured colonies on Middlebrook $7 \mathrm{H} 10$ and trypticase soy agar. Yellow to orange pigment is produced by $78 \%$ of isolates after $10-14 \mathrm{~d}$ incubation and growth occurs at 30,35 and $45^{\circ} \mathrm{C}$. Isolates grow on MacConkey agar without crystal violet and in the presence of $5 \% \mathrm{NaCl}$, are negative for arylsulfatase activity at $3 \mathrm{~d}$, and are positive for iron uptake and nitrate reductase. They produce low-level semi-quantitative catalase activity that is stable at $68{ }^{\circ} \mathrm{C}, \mathrm{pH} 7 \cdot 0$. As sole carbon sources, $95-100 \%$ of isolates utilize L-arabinose, D-glucitol (D-sorbitol), imyo-inositol, D-mannitol, L-rhamnose and D-xylose, and only 11 to $33 \%$ utilize citrate, D-galactose and/or D-trehalose. Isolates are susceptible to amikacin, ethambutol, sulfamethoxazole, intermediately susceptible to ciprofloxacin, doxycycline and tobramycin, variably susceptible to cefmetazole, cefoxitin and clarithromycin, and resistant to isoniazid and rifampin. Mycolic acids and whole-cell fatty acids are typical of mycobacterial species. By PRA, isolates exhibit a distinctive set of band patterns with $M s p \mathrm{I}$ (180, 130 and 115 bp bands), HhaI (235 and 165 bp bands), $A c i$ ( $(130,110$ and $60 \mathrm{bp}$ bands) and $B s a \mathrm{HI}$ (260, 100 and $80 \mathrm{bp}$ bands). The proposed type strain, ATCC $700504^{\mathrm{T}}\left(=\mathrm{MO} 69^{\mathrm{T}}\right)$, was recovered from a patient with a post-traumatic osteomyelitis of the calcaneus (heel).

The new species $M$. goodii and $M$. wolinskyi are clearly clinically significant, and occur in the same settings as $M$. smegmatis (sensu stricto) and members of the $M$. fortuitum complex. They are most commonly seen in post-traumatic wound infections, especially those following open fractures and with associated osteomyelitis. They differ from members of the $M$. fortuitum in the type of chronic lung disease they produce, with essentially all cases occurring in the setting of chronic lipoid pneumonia, either secondary to chronic oil ingestion or chronic aspiration (usually achalasia).

The IWGMT has had one major polyphasic study of rapidly growing mycobacteria which was published in 1972 (Kubica et al., 1972). This included nine strains which clustered with $M$. smegmatis ATCC 14468. Seven of the isolates were reference strains, and only one strain was of human origin. The current study expands this study to include a large number of human clinical $M$. smegmatis isolates and defines that they actually consist of three different species. The current study utilized more advanced genetic and chromatographic methods than were available in the 1972 study.

\section{ACKNOWLEDGEMENTS}

The authors wish to thank Michio Tsukamura, formerly of the National Chubu Hospital, Obu, Aichi, Japan; Robbie Dunlap, Denise Dunbar and Arthi Kalkura of the Texas Department of Health; Sylvia Chomyc of the Provincial Laboratory of Public Health, Edmonton, Alberta, Canada; and Vella A. Silcox from the Centers for Disease Control and Prevention, Atlanta, GA, USA, for kindly performing biochemical testing on selected isolates reported in this paper. We also thank Hans Trüper for help with the nomenclature of the new species. We also wish to thank Joanne Woodring for preparation of the manuscript.

This manuscript is dedicated to the memory of Vincent A. Steingrube, a co-author, colleague, and friend of many years, who passed away before completion of this study.

\section{REFERENCES}

Baba, T., Kaneda, K., Kusunose, E., Kusunose, M. \& Yano, I. (1988). Molecular species of mycolic acid subclasses in eight strains of Mycobacterium smegmatis. Lipids 23, 1132-1138.

Böddinghaus, B., Rogall, T., Flohr, T., Blöcker, H. \& Böttger, E. C. (1990). Detection and identification of mycobacteria by amplification of rRNA. J Clin Microbiol 28, 1751-1759.

Böttger, E. C. (1989). Rapid determination of bacterial ribosomal RNA sequences by direct sequencing of enzymatically amplified DNA. FEMS Microbiol Lett 65, 171-176.

Brown, B. A., Wallace, R. J., Jr, Onyi, G. O., De Rosas, V. \& Wallace, R. J., III (1992). Activities of four macrolides, including clarithromycin, against Mycobacterium fortuitum, Mycobacterium chelonae, and $M$. chelonae-like organisms. Antimicrob Agents Chemother 36, 180-184.

Butler, W. R. \& Kilburn, J. O. (1990). High-performance liquid chromatography patterns of mycolic acids as criteria for identification of Mycobacterium chelonae, Mycobacterium fortuitum, and Mycobacterium smegmatis. J Clin Microbiol 28, 2094-2098.

Butler, W. R., Cage, G., Desmond, E. \& 9 other authors (1996a). Standardized method for the HPLC identification of mycobacteria. Atlanta GA: Centers for Disease Control and Prevention.

Butler, W. R., Cage, G., Desmond, E. \& 9 other authors (1996b). Development of a uniform procedure for high-performance liquid chromatography (HPLC) analysis of mycobacteria and validation of a database for an automated recognition software. In Abstracts of the 96th General Meeting of the American Society 
for Microbiology, abstract U-32, p. 106. Washington, DC: American Society for Microbiology.

Domenech, P., Jimenez, M. S., Menendez, M. C., Bull, T. J., Samper, S., Manrique, A. \& Garcia, M. J. (1997). Mycobacterium mageritense sp. nov. Int $J$ Syst Bacteriol 47, 535-540.

Edwards, U., Rogal, T., Blöcker, H., Emde, M. \& Böttger, E. C. (1989). Isolation and direct sequencing of entire genes. Characterization of a gene coding for 16S ribosomal RNA. Nucleic Acids Res 17, 7843-7853.

Gordon, R. E. \& Smith, M. M. (1953). Rapidly growing, acid fast bacteria. $J$ Bacteriol 66, 41-48.

Hultman, T., Stahl, E., Hornes, E. \& Uhlen, M. (1989). Direct solid phase sequencing of genomic and plasmid DNA using magnetic bead as support. Nucleic Acids Res 17, 4937-4946.

Jost, K. C., Jr, Dunbar, D., Barth, S. S., Headley, V. L. \& Elliott, L. B. (1995). Identification of Mycobacterium tuberculosis and $M$. avium complex directly from smear-positive sputum specimens and BACTEC 12B cultures by high-performance liquid chromatography with fluorescence detection and computerdriven pattern recognition models. $J$ Clin Microbiol 33, 1270-1277.

Kasatiya, S. S., De Thokoly, I. \& Guertin, M. (1974). Mycobactéries atypiques isolées des eaux de surface au Québec. Rev Epidém Méd Soc et Santé Publ 22, 171-184.

Kazda, J., Müller, H. J., Stackebrandt, E., Daffe, M., Müller, K. \& Pitulle, C. (1992). Mycobacterium madagascariense sp. nov. Int $J$ Syst Bacteriol 42, 524-528.

Kirschner, P., Kiekenbeck, M., Meissner, D., Wolters, J. \& Böttger, E. C. (1992a). Genetic heterogeneity within Mycobacterium fortuitum complex species: genotypic criteria for identification. $J$ Clin Microbiol 30, 2772-2775.

Kirschner, P., Teske, A., Schröder, K. H., Kroppenstedt, R. M. \& Böttger, E. C. (1992b). Mycobacterium confluentis sp. nov. Int $J$ Syst Bacteriol 42, 257-262.

Kirschner, P., Meier, A. \& Böttger, E. C. (1993a). Genotypic identification and detection of mycobacteria - facing novel and uncultured pathogens. In Diagnostic Molecular Microbiology. Principles and Applications, pp. 173-190. Edited by D. H. Persing, T. F. Smith, F. C. Tenover \& T. J. White. Washington, DC: American Society for Microbiology.

Kirschner, P., Springer, B., Vogel, U., Meier, A., Wrede, A., Kiekenbeck, M., Bange, F. C. \& Böttger, E. C. (1993b). Genotypic identification of mycobacteria by nucleic acid sequence determination: report of a 2 year experience in a clinical laboratory. J Clin Microbiol 31, 2882-2889.

Kubica, G. P., Baess, I., Gordon, R. E. \& 9 other authors (1972). A co-operative numerical analysis of rapidly growing mycobacteria. $J$ Gen Microbiol 73, 55-70.

Lehmann, K. B. \& Neumann, R. O. (1931). Mycobacterium smegmatis. In Bacteriology Especially Determinative Bacteriology, pp. 755-757. New York: G. E. Stechert \& Co. (Alfred Hafner).

Levy-Frébault, V. V. \& Portaels, F. (1992). Proposed minimal standards for the genus Mycobacterium and for description of new slowly growing Mycobacterium species. Int $J$ Syst Bacteriol 42, 315-323.

Lustgarten, S. (1885). The bacillus of syphilis. Lancet 1, 609-610. Mesbah, M., Premachandran, U. \& Whitman, W. B. (1989). Precise measurement of the $\mathrm{G}+\mathrm{C}$ content of deoxyribonucleic acid by high-performance liquid chromatography. Int $J$ Syst Bacteriol 39, 159-167.

National Committee for Clinical Laboratory Standards (1990). Methods for Dilution Antimicrobial Susceptibility Tests for
Bacteria that Grow Aerobically, 2nd edn. Approved standard M7-A2. Villanova, PA: National Committee for Clinical Laboratory Standards.

Newton, J. A. Jr, Weiss, P. J., Bowler, W. A. \& Oldfield, E. C., III (1993). Soft-tissue infection due to Mycobacterium smegmatis: report of two cases. Clin Infect Dis 16, 531-533.

Pennekamp, A., Pfyffer, G. E., Wüest, J., George, C. A. \& Ruef, C. (1997). Mycobacterium smegmatis infection in a healthy woman following a facelift: case report and review of the literature. Ann Plast Surg 39, 80-83.

Pitulle, C., Dorsch, M., Kazda, J., Wolters, J. \& Stackebrandt, E. (1992). Phylogeny of rapidly growing members of the genus Mycobacterium. Int J Syst Bacteriol 42, 337-343.

Plaus, W. J. \& Hermann, G. (1991). The surgical management of superficial infections caused by atypical mycobacteria. Surgery 110, 99-103.

Richardson, A. (1970). Bovine mastitis associated with $M y c o-$ bacterium smegmatis and an untypeable Mycobacterium. Vet $\operatorname{Rec}$ 86, 497-498.

Richardson, A. (1971). The experimental production of mastitis in sheep by Mycobacterium smegmatis and Mycobacterium fortuitum. Cornell Vet 61, 640-646.

Rogall, T., Flohr, T. \& Böttger, E. C. (1982). Differentiation of Mycobacterium species by direct sequencing of amplified DNA. $J$ Gen Microbiol 136, 1915-1920.

Rogall, T., Wolters, J., Flohr, T. \& Böttger, E. C. (1990). Towards a phylogeny and definition of species at the molecular level within the genus Mycobacterium. Int $J$ Syst Bacteriol 40, 323-330.

Silcox, V. A., Good, R. C. \& Floyd, M. M. (1981). Identification of clinically significant $M y$ cobacterium fortuitum complex isolates. $J$ Clin Microbiol 14, 686-691.

Sourdis, J. \& Nei, M. (1998). Relative efficiencies of the maximum parsimony and distance-matrix methods in obtaining the correct phylogenetic tree. Mol Biol Evol 5, 298-311.

Springer, B., Böttger, E. C., Kirschner, P. \& Wallace, R. J., Jr (1995). Phylogeny of the Mycobacterium chelonae-like organism based on partial sequencing of the 16S rRNA gene and proposal of Mycobacterium mucogenicum sp. nov. Int $J$ Syst Bacteriol 45, 262-267.

Stahl, D. A. \& Urbance, J. W. (1990). The division between fastand slow-growing species corresponds to natural relationships among the mycobacteria. $J$ Bacteriol 172, 116-124.

Steingrube, V. A., Brown, B. A., Gibson, J. L., Wilson, R. W., Brown, J., Blacklock, Z., Jost, K., Ulrich, R. F. \& Wallace, R. J., Jr (1995a). DNA amplification and restriction endonuclease analysis for differentiation of 12 species and taxa of Nocardia, including recognition of four new taxa within the $N$. asteroides complex. J Clin Microbiol 33, 3096-3101.

Steingrube, V. A., Gibson, J. L., Brown, B. A., Zhang, Y., Wilson, R. W., Rajagopalan, M. \& Wallace, R. J., Jr (1995b). PCR amplification and restriction endonuclease analysis of a 65kilodalton heat shock protein gene sequence for taxonomic separation of rapidly growing mycobacteria. J Clin Microbiol 33, 149-153.

Steingrube, V. A., Wilson, R. W., Brown, B. A., Jost, K. C., Blacklock, Z., Gibson, J. L. \& Wallace, R. J., Jr (1997). Rapid identification of clinically significant species and taxa of aerobic actinomycetes, including Actinomadura, Gordona, Nocardia, Rhodococcus, Streptomyces, and Tsukamurella isolates by DNA amplification and restriction endonuclease analysis. $J$ Clin Microbiol 35, 817-822. 
Swenson, J. M., Wallace, R. J., Jr, Silcox, V. A. \& Thornsberry, C. (1985). Antimicrobial susceptibility of five subgroups of $\mathrm{Myco-}$ bacterium fortuitum and Mycobacterium chelonae. Antimicrob Agents Chemother 28, 807-811.

Telenti, A., Marchesi, F., Balz, M., Bally, F., Böttger, E. C. \& Bodmer, T. (1993). Rapid identification of mycobacteria to the species level by polymerase chain reaction and restriction enzyme analysis. J Clin Microbiol 31, 175-178.

Tønjum, T., Welty, D. B., Jantzen, E. \& Small, P. L. (1998). Differentiation of Mycobacterium ulcerans, M. marinum, and $M$. haemophilum: mapping of their relationships to $M$. $t u$ berculosis by fatty acid profile analysis, DNA-DNA hybridization, and 16S rRNA gene sequence analysis. $J$ Clin Microbiol 36, 918-925.

Tsukamura, M. (1984). Identification of mycobacteria. Obu, Aichi, Japan: Mycobacteriosis Research Laboratory of the National Chubu Hospital.

Valero-Guillén, P. L. \& Martin-Luengo, F. (1983). A gas-liquid and thin-layer chromatographic study of Mycobacterium fortuitum. Tubercle 64, 283-290.

Valero-Guillén, P. L., Martin-Luengo, F., Larsson, L. \& Jiménez, J. (1987). Demonstration of 2-methyl branched-chain fatty acids in some rapid-growing mycobacteria. FEMS Microbiol Lett 44, 303-305.

Vonmoos, S., Leuenberger, P. H., Beer, V. \& de Haller, R. (1986). Infection pleuro-pulmonaire à Mycobacterium smegmatis. Schweiz Med Wochenschr 116, 1852-1856.

Wallace, R. J., Jr, Dalovisio, J. R. \& Pankey, G. A. (1980). Disk diffusion testing of susceptibility of Mycobacterium fortuitum and Mycobacterium chelonei to antibacterial agents. Antimicrob Agents Chemother 16, 611-614.

Wallace, R. J., Jr, Nash, D. R., Tsukamura, M., Blacklock, Z. M. \& Silcox, V. A. (1988). Human disease due to Mycobacterium smegmatis. J Infect Dis 158, 52-59.

Wallace, R. J., Jr, Silcox, V. A., Tsukamura, M., Brown, B. A., Kilburn, J. O., Butler, W. R. \& Onyi, G. (1993). Clinical significance, biochemical features, and susceptibility patterns of sporadic isolates of the Mycobacterium chelonae-like organism. $J$ Clin Microbiol 31, 3231-3239.

Wayne, L. \& Kubica, G.P. (1986). Mycobacteria. In Bergey's Manual of Systematic Bacteriology, vol. 2, pp. 1436-1457. Edited by P. H. A. Sneath, N. S. Mair, M. E. Sharpe \& J. G. Holt. Baltimore: Williams \& Wilkins.

Wayne, L. G., Brenner, D. J., Colwell, R. R. \& 9 other authors (1987). International Committee on Systematic Bacteriology. Report of the ad hoc committee on reconciliation of approaches to bacterial systematics. Int J Syst Bacteriol 37, 463-464.

Wilkinson, G. T., Kelly, W. R. \& O'Boyle, D. (1982). Pyogranulomatous panniculitis in cats due to Mycobacterium smegmatis. Austr Vet $J$ 58, 77-78.

Wilson, R. W., Steingrube, V. A., Brown, B. A. \& Wallace, R. J., Jr (1998). Clinical application of PCR-restriction enzyme pattern analysis for rapid identification of aerobic actinomycete isolates. $J$ Clin Microbiol 36, 148-152.

Wolinsky, E. \& Rynearson, T. K. (1968). Mycobacteria in soil and their relation to disease-associated strains. Am Rev Respir Dis 97, 1032-1037.

Yassin, A. F., Brzezinka, H. \& Schaal, K. P. (1993). Cellular fatty acid methyl ester profiles as a tool in the differentiation of members of the genus Mycobacterium. Zentbl Bakteriol 279, 316-329.

Zhang, Y., Wallace, R. J., Jr, Steingrube, V. A., Brown, B. A., Nash, D. R., Silcox, V. A. \& Tsukamura, M. (1992). Isoelectric focusing patterns of $\beta$-lactamases in the rapidly growing mycobacteria. Tubercle Lung Dis 73, 337-344. 